\title{
Linear Kernels and Linear-Time Algorithms for Finding Large Cuts
}

\author{
Michael Etscheid $^{1}$ • Matthias Mnich ${ }^{1,2}$
}

Received: 31 March 2017 / Accepted: 13 October 2017 / Published online: 25 October 2017 C The Author(s) 2017. This article is an open access publication

\begin{abstract}
The maximum cut problem in graphs and its generalizations are fundamental combinatorial problems. Several of these cut problems were recently shown to be fixed-parameter tractable and admit polynomial kernels when parameterized above the tight lower bound measured by the size and order of the graph. In this paper we continue this line of research and considerably improve several of those results:

- We show that an algorithm by Crowston et al. (Algorithmica 72(3):734-757, 2015) for (SIGNED) MAX-CUT ABOVE EDWARDS-ERDŐs BOUND can be implemented so as to run in linear time $8^{k} \cdot O(m)$; this significantly improves the previous analysis with run time $8^{k} \cdot O\left(n^{4}\right)$.

- We give an asymptotically optimal kernel for (SIGNED) MAX-CUT ABOvE EDWARDS-ERDős BOUND with $O(k)$ vertices, improving a kernel with $O\left(k^{3}\right)$ vertices by Crowston et al. (Theor Comput Sci 513:53-64, 2013).

- We improve all known kernels for parameterizations above strongly $\lambda$-extendible properties (a generalization of the MAX- CUT results) by Crowston et al. (Proceedings of FSTTCS 2013, Leibniz international proceedings in informatics, Guwahati, 2013) from $O\left(k^{3}\right)$ vertices to $O(k)$ vertices.
\end{abstract}

Dedicated to the 60th birthday of Gregory Gutin.

Supported by ERC Starting Grant 306465 (BeyondWorstCase). An extended abstract of this work appeared in the Proc. of ISAAC 2016 [14].

$凶$ Matthias Mnich

mmnich@uni-bonn.de; m.mnich@maastrichtuniversity.nl

Michael Etscheid

etscheid@cs.uni-bonn.de

1 Institut für Informatik, Universität Bonn, Bonn, Germany

2 Department of Quantitative Economics, Maastricht University, Maastricht, The Netherlands 
- Therefore, MAX ACYCLIC SUBDIGRAPH parameterized above Poljak-Turzík bound admits a kernel with $O(k)$ vertices and can be solved in $2^{O(k)} \cdot n^{O(1)}$ time; this answers an open question by Crowston et al. (Proceedings of FSTTCS 2012, Leibniz international proceedings in informatics, Hyderabad, 2012).

All presented kernels can be computed in time $O(\mathrm{~km})$.

Keywords Max-cut · Kernelization · Linear-time algorithms

Mathematics Subject Classification 05C85 - 68R10 - 68W05

\section{Introduction}

A recent paradigm in parameterized complexity is to not only show a problem to be fixed-parameter tractable, but indeed to give algorithms with optimal run times in both the parameter and the input size. Ideally, we strive for algorithms that are linear in the input size, and optimal in the dependence on the parameter $k$ assuming a standard hypothesis such as the Exponential Time Hypothesis [22]. New results in this direction include linear-time fixed-parameter algorithms for GRAPH BIPARTIZATION [23], Planar Subgraph Isomorphism [10], DAG Partitioning [36], Planar IndePENDENT Set [11] and Subset FeEdBack Vertex Set [27].

Here, we consider the fundamental MAX-CUT problem from the view-point of linear-time fixed-parameter algorithms. In this classical NP-complete problem [24], the task is to find a bipartite subgraph of a given graph $G$ with the maximum number $\operatorname{mc}(G)$ of edges. We refer to the survey [33] for an overview of the research area.

We focus on MAX- CUT parameterized above Edwards-Erdös bound. This parameterization is motivated by the classical result of Edwards $[12,13]$ that any connected graph on $n$ vertices and $m$ edges admits a cut of size at least

$$
m / 2+(n-1) / 4
$$

This lower bound is known as the Edwards-Erdös bound, and it is tight for cliques of every odd order $n$. Ngọc and Tuza [31] gave a linear-time algorithm that finds a cut of size at least (1).

Parameterizing MAX- CUT above Edwards-Erdős bound means, for a given connected graph $G$ and integer $k$, to determine if $G$ admits a cut that exceeds (1) by an amount of $k$ : formally, the problem MAX-CUT ABOVE EDWARDS-ERDős BOUND (MAX-CUT AEE) is to determine if $\operatorname{mc}(G) \geq|E(G)| / 2+(|V(G)|-1+k) / 4$ for a given pair $(G, k)$. It was asked in a sequence of papers $[5,17,28,29]$ whether MAXCUT AEE is fixed-parameter tractable, before Crowston et al. [8] gave an algorithm that solves instances of this problem in time $8^{k} \cdot O\left(n^{4}\right)$, as well as a kernel of size $O\left(k^{5}\right)$. Their result inspired a lot of further research on this problem, leading to smaller kernels of size $O\left(k^{3}\right)$ [7] and fixed-parameter algorithms for generalizations [30] and variants [9]. 
In the SIGNED MAX-CUT problem, we are given a graph $G$ whose edges are labelled by $(+)$ or $(-)$, and we seek a maximum balanced subgraph $H$ of $G$, where balanced means that each cycle has an even number of negative edges. MAX- CUT is the special case where all edges are negative. SIGNED MAX-CUT finds applications in, e.g., modelling social networks [19], statistical physics [2], portfolio risk analysis [20], and VLSI design [4]. The dual parameterization of SIGNED MAX- CUT by the number of edge deletions was also shown to be fixed-parameter tractable [21].

Poljak and Turzík [32] showed that the property of having a large cut (i.e., a large bipartite subgraph) can be generalized to many other classical graph properties, including properties of oriented and edge-labelled graphs. They defined the notion of " $\lambda$-extendible" properties $\Pi$ and generalized the lower bound (1) to tight lower bounds for all such properties; we refer to these lower bounds as the Poljak-Turzik bound for $\Pi$. Well-known examples of such properties include bipartite subgraphs, $q$-colourable subgraphs for fixed $q$, or acyclic subgraphs of oriented graphs. Mnich et al. [30] considered the problem ABOVE POLJAK-TURZí $(\Pi)$ of finding subgraphs in $\Pi$ with $k$ edges above the Poljak-Turzik bound; they gave fixed-parameter algorithms for this problem on all "strongly" $\lambda$-extendible properties $\Pi$, thereby generalizing the algorithm for MAX- CUT. A subclass of these properties, requiring certain technical conditions, was later shown to admit polynomial kernels [9].

Gregory Gutin is one of the most prominent researchers in parameterized algorithms and complexity. In particular, he was one of the first researchers to recognise the importance of parameterizing above polynomial-time computable lower bounds on the optimal solution for maximization problems. His first result in this area, about MinIMUM LINEAR ARRANGEMENT parameterized above lower bound [16], stimulated much further research. He later contributed influential results about above-guarantee parameterizations for systems of linear equations [5], satisfiability problems [1], and constraint satisfaction [15]. In particular, he worked on fixed-parameter algorithms for finding large cuts in graphs $[7,8,17]$, the very topic of this article. We thus happily dedicate this work to Gregory Gutin on the occasion of his 60th birthday.

\subsection{Our Contributions}

Linear-Time FPT Our first result shows that the fixed-parameter algorithm by Crowston et al. [7] for the SIGNED MAX- CUT AEE problem can be implemented so as to run in linear time:

Theorem 1 (SIGNED) MAX- CUT AEE can be solved in time $8^{k} \cdot O(m)$.

Theorem 1 considerably improves the earlier run time analysis [7,8], which shows a run time of $8^{k} \cdot O\left(n^{4}\right)$. At the same time, our algorithm improves the very involved algorithm by Bollobás and Scott [3] that considers the weaker lower bound $m / 2+(\sqrt{8 m+1}-1) / 8$ instead of (1). Third, Theorem 1 generalizes the linear-time algorithm by Ngọ and Tuza [31] for the special case of MAX-CUT with $k=0$. Note that MAX-CUT AEE cannot be solved in time $2^{o(k)} \cdot n^{O(1)}$ assuming the Exponential Time Hypothesis [8]. 
Linear Vertex Kernels Our second contribution is a kernel with a linear number $O(k)$ of vertices for MAX-CUT AEE and its generalization SIGNED MAX-CUT AEE.

Theorem 2 The (SIGNED) MAX-CUT AEE problem admits a kernel with $O(k)$ vertices, which can be computed in time $O(\mathrm{~km})$.

These results considerably improve the previous best kernel bound of $O\left(k^{3}\right)$ vertices by Crowston et al. [7]. Moreover, the presented kernel completely resolves the asymptotic kernelization complexity of (SIGNED) MAX-CUT AEE, since a kernel with $o(k)$ vertices would again contradict the Exponential-Time Hypothesis, as the MAX-CUT problem can be solved by checking all vertex bipartitions. On top of that, our kernelization is also fast. In fact, we only need to compute $O(k)$ DFS/BFS trees. The rest of the algorithm runs in time $O(m)$.

Extensions to Strongly $\lambda$-Extendible Properties As mentioned, the property of graphs having large bipartite subgraphs can be generalized to $\lambda$-extendible properties as defined by Poljak and Turzík [32] (we defer the formal definitions to Sect. 2). For a given $\lambda$-extendible property $\Pi$, we consider the following problem:

ABOve POLJAK- Turzík $(\Pi)$

Input: A connected graph $G$ and an integer $k$.

Question: Does $G$ have a spanning subgraph $H \in \Pi$ s.t. $|E(H)| \geq \lambda \cdot|E(G)|+$ $\frac{1-\lambda}{2} \cdot(|V(G)|-1)+k$ ?

MAX-CUT AEE is a special case of this problem with $\lambda=\frac{1}{2}$. Note the slight change in the definition of $k$ compared to (SIGNED) MAX-CUT AEE, where $k$ was divided by $4=\frac{2}{1-\lambda}$ for $\lambda=\frac{1}{2}$.

Crowston et al. [7] gave polynomial kernels for ABOve POLJAK-TuRZíK( $\Pi$ ), for all strongly $\lambda$-extendible properties $\Pi$ on possibly oriented and/or labelled graphs satisfying at least one of the following properties:

(P1) $\lambda \neq \frac{1}{2}$; or

(P2) $G \in \Pi$ for all graphs $G$ whose underlying simple graph is $K_{3}$; or

(P3) $\Pi$ is a hereditary property of simple or oriented graphs.

Their kernels have $O\left(k^{3}\right)$ or $O\left(k^{2}\right)$ vertices, depending on the exact problem.

Our third result improves all these kernels for strongly $\lambda$-extendible properties to asymptotically optimal $O(k)$ vertices:

Theorem 3 Let $\Pi$ be any strongly $\lambda$-extendible property of (possibly oriented and/or labelled) graphs satisfying (P1), or (P2), or (P3). Then ABOVE POLJAK-TuRZíK( $\Pi)$ admits a kernel with $O(k)$ vertices, which is computable in time $O(\mathrm{~km})$.

Consequences for Acyclic Subdigraphs Theorem 3 has several applications. For instance, Raman and Saurabh [34] asked for the parameterized complexity of the MAX ACYCLIC SUBDIGRAPH problem above the Poljak-Turzík bound: Given a weakly connected oriented graph $G$ on $n$ vertices and $m$ arcs, does it have an acyclic sub-digraph of at least $m / 2+(n-1) / 4+k$ arcs? For this problem, Crowston et al. [6] gave an 
algorithm with run time $2^{O(k \log k)} \cdot n^{O(1)}$ and showed a kernel with $O\left(k^{2}\right)$ vertices. They explicitly asked whether the kernel size can be improved to $O(k)$ vertices, and whether the run time can be improved to $2^{O(k)} \cdot n^{O(1)}$. Here, we answer their questions in the affirmative by using Theorem 3 and then applying an $O^{*}\left(2^{n}\right)$-time algorithm by Raman and Saurabh [35, Thm. 2] to our kernel with $O(k)$ vertices.

Corollary 1 MAX ACYCLIC SUBDIGRAPH parameterized above Poljak-Turzík bound admits a kernel with $O(k)$ vertices and can be solved in time $2^{O(k)} \cdot n^{O(1)}$.

Again, assuming the Exponential Time Hypothesis, the run time of this algorithm is asymptotically optimal (by a standard reduction from VERTEX COVER).

This work is organized as follows. After the Preliminaries in Sect. 2, we discuss in Sect. 3 that SIGNED MAX-CUT AEE can be solved in linear time, i.e., we show Theorem 1. Section 4 is dedicated to SIGNED MAX-CUT AEE kernelization resulting in Theorem 2. Section 5 turns to the generalization to $\lambda$-extendible properties, showing Theorem 5. A short discussion in Sect. 6 concludes the paper.

\section{Preliminaries}

We use $\uplus$ to denote the disjoint union of sets. The term "graph" refers to finite undirected graphs without self-loops, parallel edges, edge directions, or labels. For a graph $G$, let $V(G)$ denote its set of vertices and let $E(G)$ denote its set of edges. In an oriented graph, each edge $e=\{u, v\}$ has one of two directions, $\vec{e}=(u, v)$ and $\overleftarrow{e}=(v, u)$; thus, an oriented graph is a digraph without 2-cycles and loops. Distinct vertices $a, b, c$ are said to induce a triangle $(a, b, c)$ if they form a complete subgraph. In a labelled graph, each edge in $E(G)$ receives one of a constant number of labels. For an oriented and/or labelled graph $G$, let $\langle G\rangle$ denote the underlying simple graph obtained from omitting orientations and/or labels. Throughout the paper, we assume graphs to be encoded as adjacency lists.

A graph is connected if there is a path between any two of its vertices. A connected component of $G$ is a maximal connected subgraph of $G$. A cut vertex of a graph $G$ is a vertex whose removal increases the number of connected components. A graph is 2-connected if it does not contain any cut vertices. A maximal 2-connected subgraph of a graph $G$ is called a block of $G$. A block that contains at most one cut vertex of $G$ is called a leaf block of $G$. A clique tree is a connected graph whose blocks are cliques, where a clique is a complete subgraph of a graph. A clique forest is a graph whose connected components are clique trees. ${ }^{1}$ For an oriented and/or labelled graph $G$ we say that $G$ has one of the above-defined properties if $\langle G\rangle$ does.

Let $G$ be a graph. For a vertex subset $X \subset V(G)$, the (vertex-)induced subgraph $G[X]$ is the graph with vertex set $X$ whose edge set consists of all the edges of $G$ with both endpoints in $X$. Similarly, we define $G-X=G[V(G) \backslash X]$ for a vertex subset $X \subseteq V(G)$ and $G-x=G-\{x\}$ for a vertex $x \in V(G)$.

For a vertex $v \in V(G)$, let $N_{G}(v)=\{u \in V(G) \mid\{u, v\} \in E(G)\}$. For signed graphs $G$, we define $N_{G}(v)=N_{\langle G\rangle}(v)$. For a vertex set $V^{\prime} \subseteq V(G)$, let $N_{G}\left(V^{\prime}\right)=$

\footnotetext{
${ }^{1}$ Clique forests are sometimes called block graphs; however, there are competing definitions for this term in the literature and so we refrain from using it.
} 
$\left(\bigcup_{v \in V^{\prime}} N_{G}(v)\right) \backslash V^{\prime}$. For disjoint vertex sets $V_{1}, V_{2} \subseteq V(G)$, let $E\left(V_{1}, V_{2}\right)$ denote the set of edges with one endpoint in $V_{1}$ and the other endpoint in $V_{2}$. For a signed graph $G$, let $E^{+}(G) \subseteq E(G)$ be the edges with positive labels, and $E^{-}(G)=E(G) \backslash E^{+}(G)$ be the edges with negative labels. Define $N_{G}^{+}(v)=\left\{u \in V(G) \mid\{v, u\} \in E^{+}(G)\right\}$ and $N_{G}^{-}(v)=\left\{u \in V(G) \mid\{v, u\} \in E^{-}(G)\right\}$ for all $v \in V(G)$. A sequence of vertices $\left(v_{0}, v_{1}, \ldots, v_{\ell}\right)$ is a path in $G$ if $v_{0}, v_{1}, \ldots, v_{\ell}$ are distinct vertices of $G$ and $\left\{v_{i}, v_{i+1}(\bmod \ell)\right\} \in E(G)$ for $i=0, \ldots, \ell$. For vertices $u, v \in V(G)$, a $[u, v]$-path is a path in $G$ between $u$ and $v$. A path is induced if additionally $\left\{v_{i}, v_{j}\right\} \notin E(G)$ for $i=0, \ldots, \ell$ and $j \neq i+1(\bmod \ell)$. The length of a path is the number of edges it contains, and an $\ell$-path is a path of length $\ell$.

A graph property $\Pi$ is simply a set of graphs. For a graph $G$, a $\Pi$-subgraph is a subgraph of $G$ that belongs to $\Pi$. A graph property $\Pi$ is hereditary if for any $G \in \Pi$ also all vertex-induced subgraphs of $G$ belong to $\Pi$. Poljak and Turzík [32] defined the notion of " $\lambda$-extendibility" for graph properties $\Pi$, and proved a lower bound on the size of any $\Pi$-subgraph in arbitrary graphs. A related notion of "strong $\lambda$ extendibility" was introduced by Mnich et al. [30]; any strongly $\lambda$-extendible property is $\lambda$-extendible, but it is unclear whether the other direction holds.

Definition 1 Let $\mathcal{G}$ be a class of (possibly labelled and/or oriented) graphs and let $\lambda \in(0,1)$. A (graph) property $\Pi$ is strongly $\lambda$-extendible on $\mathcal{G}$ if it satisfies the following properties:

(i) inclusiveness: $\left\{G \in \mathcal{G} \mid\langle G\rangle \in K_{1}, K_{2}\right\} \subseteq \Pi$.

(ii) block additivity: $G \in \mathcal{G}$ belongs to $\Pi$ if and only if each block of $G$ belongs to $\Pi$.

(iii) extendibility: For any $G \in \mathcal{G}$ and any partition $U \uplus W$ of $V(G)$ for which $G[U], G[W] \in \Pi$ there is a set $F \subseteq E(U, W)$ of size $|F| \geq \lambda|E(U, W)|$ for which $G-(E(U, W) \backslash F) \in \Pi$.

The set of all bipartite graphs $\Pi_{\text {bipartite }}$ is a strongly $\frac{1}{2}$-extendible property. Thus, MAX-CuT AEE is equivalent to ABOve POLJAK-Turzík BOUnd $\left(\Pi_{\text {bipartite }}\right)$.

Poljak and Turzík [32] showed that, given a (strongly) $\lambda$-extendible property $\Pi$, any connected graph $G$ contains a subgraph $H \in \Pi$ with at least $\lambda|E(G)|+$ $\frac{1-\lambda}{2}(|V(G)|-1)$ edges. We denote this lower bound by $\operatorname{pt}(G)$. Further, we define the excess of $G$ over this lower bound with respect to $\Pi$ as $\operatorname{ex}(G)=\max \{|E(H)|-$ $\operatorname{pt}(G) \mid H \subseteq G, H \in \Pi\}$. When considering properties of labelled and/or oriented graphs, we denote by $\operatorname{ex}\left(K_{t}\right)$ the minimum value of $\operatorname{ex}(G)$ over all labelled and/or oriented graphs $G$ with $\langle G\rangle=K_{t}$; here, $K_{t}$ denotes the complete graph of order $t$.

A strongly $\lambda$-extendible property $\Pi$ diverges on cliques if $\operatorname{ex}\left(K_{j}\right)>\frac{1-\lambda}{2}$ for some $j \in \mathbb{N}$. For example, every strongly $\lambda$-extendible property with $\lambda \neq \frac{1}{2}$ diverges on cliques [9]. We recall the following fact about diverging properties:

Proposition 1 [9, Lemmas 7-8] Let $\Pi$ be a strongly $\lambda$-extendible property diverging on cliques, and let $j \in \mathbb{N}, a>0$ be such that $\operatorname{ex}\left(K_{j}\right)=\frac{1-\lambda}{2}+a$. Then $\operatorname{ex}\left(K_{i}\right) \geq r a$ for all $r \in \mathbb{N}$ and $i \geq r j$.

We need the following proposition in all sections. For SIGNED MAX- CUT, we will apply it with $\lambda=\frac{1}{2}$. 
Proposition 2 [9, Lemma 6] Let $\Pi$ be a strongly $\lambda$-extendible property, let $G$ be a connected graph and let $U_{1} \uplus U_{2}$ be a partition of $V(G)$ into non-empty sets $U_{1}, U_{2}$. For $i \in\{1,2\}$ let $c_{i}$ be the number of connected components of $G\left[U_{i}\right]$. If $\operatorname{ex}\left(G\left[U_{i}\right]\right) \geq k_{i}$ for some $k_{i} \in \mathbb{R}$ and $i \in\{1,2\}$, then $\operatorname{ex}(G) \geq k_{1}+k_{2}-\frac{1-\lambda}{2}\left(c_{1}+c_{2}-1\right)$.

\section{A Linear-Time Fixed-Parameter Algorithm for Signed Max-Cut AEE}

In this section we consider the SIGNED MAX-CUT AEE problem. We show that the fixed-parameter algorithm given by Crowston et al. [7] can be implemented so as to run in time $8^{k} \cdot O(|E(G)|)$. That is, given a connected graph $G$ whose edges are labelled either positive $(+)$ or negative $(-)$, and an integer $k$, we can decide in time $8^{k} \cdot O(|E(G)|)$ whether $G$ has a balanced subgraph with at least $|E(G)| / 2+$ $(|V(G)|-1+k) / 4$ edges. This will prove Theorem 1 .

We build on the following classical characterization of signed graphs:

Proposition 3 (Harary [18]) A signed graph $G$ is balanced if and only if there exists a partition $V_{1} \uplus V_{2}=V(G)$ such that all edges in $G\left[V_{1}\right]$ and $G\left[V_{2}\right]$ are positive and all edges $E\left(V_{1}, V_{2}\right)$ between $V_{1}$ and $V_{2}$ are negative.

The algorithm by Crowston et al. [7] starts by applying the following seven reduction rules. We restate them here, as they are crucial for our results. A reduction rule is 1 safe if, on input $(G, k)$ it returns a pair $\left(G^{\prime}, k^{\prime}\right)$ such that $(G, k)$ is a "yes"-instance for SIGNED MAX-CUT AEE if $\left(G^{\prime}, k^{\prime}\right)$ is. (Note that the converse direction does not have to hold). In a signed graph $G$ we call a triangle positive if its number of negative edges is even. In the description of the rules, $G$ is always a connected signed graph and $C$ is always a clique without positive triangles.

We initialize an empty set $S$ of marked vertices. (Note: In previous work the term selected vertices was also used, so we stick to the set name $S$ ).

Reduction Rule 1 If $(a, b, c)$ is a positive triangle such that $G-\{a, b, c\}$ is connected, add $a, b, c$ to $S$ and delete them from $G$, and set $k^{\prime}=k-3$.

Reduction Rule 2 If $(a, b, c)$ is a positive triangle such that $G-\{a, b, c\}$ has exactly two connected components $C$ and $Y$, then add $a, b, c$ to $S$ and delete them from $G$, delete $C$, and set $k^{\prime}=k-2$.

Reduction Rule 3 Let $C$ be a connected component of $G-v$ for some vertex $v \in$ $V(G)$. If there exist $a, b \in V(C)$ such that $G-\{a, b\}$ is connected and there is an edge $\{a, v\}$ but no edge $\{b, v\}$, then add $a, b$ to $S$ and delete them from $G$, and set $k^{\prime}=k-2$.

Reduction Rule 4 Let $C$ be a connected component of $G-v$ for some vertex $v \in$ $V(G)$. If there exist $a, b \in V(C)$ such that $G-\{a, b\}$ is connected and $(a, b, v)$ is $a$ positive triangle, then add $a, b$ to $S$ and delete them from $G$, and set $k^{\prime}=k-4$.

Reduction Rule 5 If there is a vertex $v \in V(G)$ such that $G-v$ has a connected component $C$ such that $G[V(C) \cup\{v\}]$ is a clique without positive triangles, then delete $C$. If $|V(C)|$ is odd, set $k^{\prime}=k-1$; otherwise, set $k^{\prime}=k$. 
Reduction Rule 6 If $a, b, c \in V(G)$ induce a 2-path $(a, b, c)$ such that $G-\{a, b, c\}$ $i$ connected, then add $a, b, c$ to $S$ and delete them from $G$, and set $k^{\prime}=k-1$.

Reduction Rule 7 Let $C, Y$ be the connected components of $G-\{v, b\}$ for some $v, b \in$ $V(G)$ such that $\{v, b\} \notin E(G)$. If $G[V(C) \cup\{v\}]$ and $G[V(C) \cup\{b\}]$ are cliques without positive triangles, then add $v, b$ to $S$ and delete them from $G$, delete $C$, and set $k^{\prime}=k-1$.

Note: Rules 1/2/4 require positive edges. Hence, the other four rules suffice to handle the classical MAX-CUT AEE problem, where all edges are negative. We will make use of this in Sect. 5.

We will call the vertex $v$ of Rule 5 the anchor of the removed vertex set $V(C)$.

We slightly changed Rule 5. Crowston et al. [7] always set $k^{\prime}=k$, whereas we set $k^{\prime}=k-1$ when $|V(C)|$ is odd. In this case, $\operatorname{pt}(G[V(C) \cup\{v\}])$ cannot be integral because $|V(C) \cup\{v\}|$ is even, and thus $\operatorname{ex}(G[V(C) \cup\{v\}]) \geq \frac{1}{4}$. Therefore, our change for $k$ is 1 -safe by the following result.

Proposition 4 [7, Lemma 2] Let $G$ be a connected signed graph and let $Z$ be a connected component of $G-v$ for some $v \in V(G)$. Then $\operatorname{ex}(G)=\operatorname{ex}(G-Z)+$ $\operatorname{ex}(G[V(Z) \cup\{v\}])$.

We subsume the results by Crowston et al. [7] in the following proposition.

Proposition 5 [7] Rules 1-7 are 1-safe. To any connected signed graph $G$ with at least one edge, one of these rules applies and the resulting graph is connected. For the set $S$ of vertices marked during the exhaustive application of Rules 1-7, $G-S$ is a clique forest. If $|S|>3 k$, then $(G, k)$ is a "yes"-instance for SIGNED MAX-CUT AEE.

Following Crowston et al. [7, Corollary 3], we assume-without loss of generalityfrom now on that the resulting clique forest $G-S$ does not contain positive edges.

Lemma 1 Let $G$ be a signed graph for which $\langle G\rangle$ is a complete graph. Then in time $O(|E(G)|)$, we either find a positive triangle in $G$ or decide that none exists.

Proof Let $H=\left(V(G), E^{+}(G)\right)$, where $E^{+}(G)$ are the positive edges in $G$. As a positive triangle has either exactly zero or exactly two negative edges, our task is to find either a triangle in $H$ or an edge $\{a, b\} \in E(H)$ and a vertex $c \in V(H)$ such that $\{a, c\},\{b, c\} \notin E(H)$ (remember that $\langle G\rangle$ is a complete graph). In order to achieve this, we try to find a 2-colouring, i.e., a bipartition, of $H$ using breadth-first search [26].

- If this succeeds, then we have found a bipartition $A \uplus B$ of $V(H)$ such that $H[A], H[B]$ are edgeless. If $H$ is a complete bipartite graph or $E(H)=\emptyset$, then $G$ does not contain a positive triangle. Otherwise, we can assume, without loss of generality, that there is a vertex $a \in A$ with $\emptyset \neq N_{H}(a) \neq B$, i.e., there are vertices $b \in N_{H}(a)$ and $c \in B \backslash N_{H}(a)$. Then $(a, b, c)$ is a positive triangle.

- If it fails, then we have found an odd cycle $C=\left(x_{1}, \ldots, x_{\ell}, x_{1}\right)$, i.e., $\ell$ is odd. If $\left\{x_{1}, x_{3}\right\} \in E(G)$, then $\left(x_{1}, x_{2}, x_{3}\right)$ is a positive triangle in $G$. Otherwise, if $\left\{x_{1}, x_{3}\right\} \notin E(G)$ and $\left\{x_{1}, x_{4}\right\} \notin E(G)$, then $\left(x_{1}, x_{3}, x_{4}\right)$ is a positive triangle in $G$. 
Otherwise, $\left(x_{1}, x_{4}, \ldots, x_{\ell}, x_{1}\right)$ is an odd cycle in $H$ with length smaller than $C$. Repeat this procedure until a triangle is found. Note that every iteration can be performed in constant time.

Hence, in linear time we either find a positive triangle or decide that none exists.

Definition 2 Let $T$ be a DFS tree of a graph $G$ rooted at a vertex $r \in V(G)$. For two vertices $v, w \in G$, we say that $v$ is lower than $w$ if its distance to $r$ with respect to $T$ is larger than the distance from $w$ to $r$ with respect to $T$. For a vertex $v$, we denote by $T_{v}$ the subtree of $T$ rooted at $v$. A child tree of a vertex $v$ is the subtree $T_{w}$ of a child $w$ of $v$.

Lemma 2 Let $G$ be a 2-connected graph and let $r \in V(G)$ such that $G-r$ is not 2-connected. Then in time $O(|E(G)|)$, we can find an induced 2-path $P$ in $G-r$ such that $G-V(P)$ is connected.

Proof Note that $r \in V(G-V(P))$.

We first state the algorithm before we discuss why it is well-defined and correct.

1. Compute a cut vertex $v$ of $G-r$ and let $Z_{1}, Z_{2}$ be 2-connected components of $G-r$ containing $v$.

2. For $i \in\{1,2\}$, find a vertex $u_{i}$ of $V\left(Z_{i}\right) \backslash\{v\}$ with minimum distance to $r$ with respect to $G-v$, and let $P_{i}$ be a shortest $\left[r, u_{i}\right]$-path in $G-v$.

3. For $i \in\{1,2\}$, let $T_{i}$ be a DFS tree of $Z_{i}$ rooted at $v$ such that $u_{i}$ is a child of $v$ in $T_{i}$ if $\left\{v, u_{i}\right\} \in E(G)$.

4. For $i \in\{1,2\}$, let $w_{i}$ be a lowest (w.r.t. $T_{i}$ ) neighbour of $v$ in $Z_{i}$.

5. Return the induced 2-path $P=\left(w_{1}, v, w_{2}\right)$.

Because $G-r$ is not 2-connected, a cut vertex $v$ and thus also $Z_{1}$ and $Z_{2}$ exist. The paths $P_{1}$ and $P_{2}$ exist because $G$ is 2-connected, i.e., $G-v$ is still connected. As $w_{1}$ and $w_{2}$ are in different 2-connected components of $G-r$, they are in different connected components of $G-\{r, v\}$ and therefore not adjacent. Hence, $P$ is indeed an induced path and the algorithm is well-defined.

We now prove that $G-V(P)$ is connected by showing that for every $x \in V(G)-$ $V(P)$ there is an $[x, r]$-path in $G-V(P)$. Note that $r$ is still contained in $G-V(P)$ because it is by definition of $Z_{1}$ and $Z_{2}$ not contained in either of them.

- First look at the case that $x \in V\left(Z_{1}\right)$ (the case $x \in V\left(Z_{2}\right)$ is analogous). This implies $\left|V\left(Z_{1}\right)\right| \geq 3$ because two vertices of $Z_{1}$ are contained in $P$. Because $Z_{1}$ is 2-connected, the vertex $v$ is adjacent to at least two vertices of $V\left(Z_{1}\right) \backslash\{v\}$. It follows that $u_{1}$ cannot be the lowest neighbour of $v$ in $Z_{1}$ by construction of $T_{1}$ and thus $u_{1}$ is contained in $G-V(P)$. Because $v$ is a cut vertex of $G-r$, every path from $r$ to $Z_{1}$ that uses a vertex from $Z_{2}$ must also use $v$. But $P_{1} \subseteq G-v$, i.e., $P_{1}$ cannot use vertices from $Z_{2}$ and thus it does not contain $w_{2}$. Hence, $P_{1} \subseteq G-V(P)$ and therefore $r$ is connected to a vertex from $Z_{1}-\left\{v, w_{1}\right\}$ (namely, $u_{1}$ ).

Because $w_{1}$ is the lowest (w.r.t. $T_{1}$ ) neighbour of $v$ in $Z_{1}$, every child tree of $w_{1}$ is not adjacent to $v$. But because $Z_{1}$ is 2-connected, every child tree of $w_{1}$ is adjacent to a vertex that is higher than $w_{1}$ as otherwise $w_{1}$ would be a cut vertex of $Z_{1}$. This shows that $Z_{1}-\left\{v, w_{1}\right\}$ is connected and thus there is an $[x, r]$-path in $G-V(P)$ via $u_{i}$. 
- Now consider the remaining case that $x$ is neither contained in $Z_{1}$ nor in $Z_{2}$. As $G$ is 2-connected, there are two $[x, r]$-paths $Q_{1}, Q_{2}$ in $G$ that do not share an internal vertex. Let $y$ be the vertex of $V\left(Z_{1}\right) \cup V\left(Z_{2}\right)$ that is nearest to $x$ with respect to $G-r$. Because $G-r$ is not 2-connected and $Z_{1}$ as well as $Z_{2}$ are 2-connected components of $G-r$, the vertex $y$ is a cut vertex of $G-r$ separating $x$ from $V\left(Z_{1}\right) \cup V\left(Z_{2}\right) \backslash\{y\}$. This means that every $[x, r]$-path that uses vertices from $V\left(Z_{1}\right) \cup V\left(Z_{2}\right)$ must also contain the vertex $y$. Hence, as $Q_{1}$ and $Q_{2}$ do not share internal vertices, only at most one of these two paths can use vertices from $V\left(Z_{1}\right) \cup V\left(Z_{2}\right)$ and thus one of the paths $Q_{1}, Q_{2}$ is fully contained in $G-V(P)$.

Finally we show that the algorithm runs in linear time. The vertex $v$ and the 2-connected components $Z_{1}, Z_{2}$ can be found in time $O(|E(G)|)$ using any linear-time algorithm for finding 2-connected components in undirected graphs. The vertices $u_{1}, u_{2}$ and the paths $P_{1}, P_{2}$ can be found via breadth-first search in $G-v$, starting in $r$. The DFS trees $T_{1}, T_{2}$ can also be computed in linear time. The restriction that $u_{i}$ shall be the direct child of $v$ if these two vertices are adjacent, can easily be followed by selecting the edge $\left\{v, u_{i}\right\}$ as the first traversed edge in the depth-first search. In linear time, we can find the neighbour $w_{i}$ of $v$ that is the lowest with respect to $T_{i}, i \in\{1,2\}$. This completes the proof.

Lemma 3 Let $G$ be a connected signed graph, let $X$ be a leaf block of $G$, and let $r \in$ $V(G)$ such that $V(X) \backslash\{r\}$ does not contain any cut vertex of $G$. Then we can always apply one of Rules 1-7 to $G$ such that only vertices from $X$ are marked and deleted, in time $O(|E(X)|)$.

Proof Let us first argue why we may assume for this proof that the edges of $X$ are given in form of an adjacency matrix (this is a standard argument):

Let $v_{1}, \ldots, v_{n^{\prime}}$ be the vertices and $e_{1}, \ldots, e_{m^{\prime}}$ be the edges of $X$. Create an array $L$ of size $m^{\prime}$ and a 2-dimensional array $M$ of size $n^{\prime} \times n^{\prime}$, both of which are not initialized and therefore need only constant construction time. For every edge $e_{i}=\left\{v_{a}, v_{b}\right\}$, where $a<b$, set $L[i]$ to $(a, b)$ and set $M[a][b]$ to $i$. This takes $O\left(m^{\prime}\right)$ time in total. After it, $L$ is completely and $M$ is partly initialized.

In order to check now in constant time whether an edge $\{a, b\}, a<b$, exists in $X$, try to read the integer $i$ that is stored in $M[a][b]$. Then $M[a][b]$ is initialized and thus the edge $\{a, b\}$ exists if and only if $L[i]$ is set to $(a, b)$.

Having this construction, we can check in time $O(|E(X)|)$ whether a subset $X^{\prime} \subseteq$ $V(X)$ induces a clique in $G$ in the following way: We test for the $O\left(\left|X^{\prime}\right|^{2}\right)$ many pairs of vertices of $X^{\prime}$ in lexicographically ascending order whether the corresponding edge exists in $X$. We stop at the first vertex pair that does not exist as edge. This way we check at most $|E(X)|+1=O(|E(X)|)$ pairs and every check can be processed in constant time.

Let us now turn to the proof of the lemma. We consider the following cases:

1. If $X$ is a clique, then we can find with Lemma 1 in time $O(|E(X)|)$ a positive triangle $(a, b, c)$ in $X$ or decide that none exists. If none exists, then Rule 5 applies to $X-r$ and $r$. If $G-\{a, b, c\}$ is connected, then Rule 1 applies to $(a, b, c)$. 
If $G-\{a, b, c\}$ is not connected, then Rule 2 applies if $r \notin\{a, b, c\}$, and Rule 4 applies if $r \in\{a, b, c\}$.

2. If $X$ is not a clique, but $X-r$ is a clique, then again try to find a positive triangle $(a, b, c)$ in $X-r$. If this fails, then Rule 3 applies. Otherwise, $r \notin\{a, b, c\}$ and thus Rule 1 or Rule 2 applies.

3. If $X$ is not a clique, $N_{X}(r)=\{x, y\}$ for some vertices $x, y \in V(X)$ with $\{x, y\} \notin$ $E(X)$, and $X-\{r, x\}$ as well as $X-\{r, y\}$ are cliques, then again try to find a positive triangle $(a, b, c)$ in $X-\{r, x\}$ or $X-\{r, y\}$. If this fails, then Rule 7 applies. Otherwise, $r \notin\{a, b, c\}$ and thus Rule 1 or Rule 2 applies.

4. Now assume that none of the previous cases applies. If $X-r$ is not 2-connected, then we can find with Lemma 2 a path $P$ in $X$ that does not use $r$ such that $X-V(P)$ and thus also $G-V(P)$ is connected. Hence, Rule 6 applies to $P$. From now on we assume that $X-r$ is 2-connected.

We perform a breadth-first search on $X$ starting in $r$ to compute the distance from $r$ to all vertices $x \in V(X) \backslash\{r\}$. For $i \geq 1$, let $L_{i} \subseteq V(X) \backslash\{r\}$ be the set of vertices with distance $i$ to $r$.

Find vertices $x, y \in V(X) \backslash\{r\}$ such that $\{x, y\} \notin E(X), L_{1} \neq\{x, y\}$, and the distance from $r$ to $x$ is minimum. We do this again by testing all possible vertex pairs in lexicographically ascending order. After at most $|E(X)|$ tested pairs, two non-adjacent vertices must have been found. Note that these vertices must exist, as otherwise one of the previous cases would be applicable.

Find a shortest path $Q$ from $r$ to $x$; by breadth-first search, this can be done in time $O(|E(X)|)$. The length of $Q$ is at most 2, because if $L_{3} \neq \emptyset$, then every pair of vertices from $L_{1}$ and $L_{3}$ is non-adjacent.

Then we try to find via breadth-first search in time $O(|E(X)|)$ a shortest path $P$ from $x$ to $y$ in $X-(V(Q) \backslash\{x\})$. If $P$ exists, then $P$ is an induced path. Let $P^{\prime}$ be the unique connected subgraph of $P$ containing $x$ with $\left|V\left(P^{\prime}\right)\right|=3$ (i.e., $P^{\prime}$ contains the "first" three vertices of $P$ ).

If $P^{\prime}$ exists and $G-V\left(P^{\prime}\right)$ is connected, then Rule 6 applies. Otherwise, we have found a (not necessarily induced) path $\left(p_{1}, \ldots, p_{\ell}\right)$ of vertices from $X\left[V(Q) \cup V\left(P^{\prime}\right)\right]$ with $p_{1}=r$ such that $G\left[X-\left\{p_{1}, \ldots, p_{\ell}\right\}\right]$ is not connected. By construction, it holds that $\ell \leq 6$. As $X$ is 2-connected, there is an $i \in\{0, \ldots, \ell-1\}$ such that $X^{\prime}:=X \backslash\left\{p_{1}, \ldots, p_{i}\right\}$ is 2-connected, but $X^{\prime} \backslash\left\{p_{i+1}\right\}$ is not. Using Lemma 2, we can find a vertex-induced path $P^{\prime}$ in $X^{\prime}$ that does not use $p_{i+1}$ such that $X^{\prime}-V\left(P^{\prime}\right)$ is connected. In particular, every vertex is reachable from $p_{i+1}$ and thus from $p_{1}=r$ in $X-V\left(P^{\prime}\right)$. It follows that $G-V\left(P^{\prime}\right)$ is connected and Rule 6 applies to $P^{\prime}$.

Given an instance $(G, k)$, we can thus compute in time $O(k \cdot|E(G)|)$ a vertex set $S$ that either proves that $(G, k)$ is a "yes"-instance or $G-S$ is a clique forest. We now show that, if a partition for the vertices in $S$ is already given, we can in time $O(|E(G)|)$ compute an optimal extension to $G$. We use the following problem, which goes back to Crowston et al. [8]: 
MAX- CUT EXTENSION

Input: A clique forest $G_{S}$ and weight functions $w_{0}, w_{1}: V\left(G_{S}\right) \rightarrow \mathbb{N}_{0}$.

Task: Find an assignment $\varphi: V\left(G_{S}\right) \rightarrow\{0,1\}$ maximizing $\sum_{\{x, y\} \in E\left(G_{S}\right)}|\varphi(x)-\varphi(y)|+\sum_{i=0}^{1} \sum_{x: \varphi(x)=i} w_{i}(x)$.

Lemma 4 MAX- CUT EXTENSION can be solved in time $O\left(\left|V\left(G_{S}\right)\right|+\left|E\left(G_{S}\right)\right|\right)$ on clique forests $G_{S}$.

Proof In order to solve MAX-CUT EXTENSION on $G_{S}$ in time $O\left(\left|V\left(G_{S}\right)\right|+\left|E\left(G_{S}\right)\right|\right)$, we use the natural approach suggested by Crowston et al. [8], and argue why it runs in the desired time. We provide a transformation that replaces an instance $I=\left(G_{S}, w_{0}, w_{1}\right)$ with an equivalent instance $I^{\prime}=\left(G_{S}^{\prime}, w_{0}^{\prime}, w_{1}^{\prime}\right)$ such that $G_{S}^{\prime}$ has fewer blocks than $G_{S}$, and that we can recover an optimal solution for $I$ from an optimal solution for $I^{\prime}$. By repeatedly applying the transformation we obtain a trivial instance, and thus the optimal solution for $I$.

We may assume that $G_{S}$ is connected, as otherwise we can handle each connected component of $G_{S}$ separately. Let $X \cup\{r\}$ be the vertices of a leaf block in $G$, with $r$ a cut vertex of $G_{S}$ (unless $G_{S}$ consists of a single block, in which case let $r$ be an arbitrary vertex and $\left.X=V\left(G_{S}\right) \backslash\{r\}\right)$. Recall that by definition of a clique forest, $X \cup\{r\}$ is a clique. For each possible assignment to $r$, we will calculate the optimal extension to the vertices in $X$. (This optimal extension depends only on the assignment to $r$, since no other vertices are adjacent to vertices in $X$.) We can then remove all vertices in $X$, and change the values of $w_{0}(r)$ and $w_{1}(r)$ to reflect the optimal extension for each assignment.

Suppose we assign $r$ the value 1 . Let $\varepsilon(x)=w_{1}(x)-w_{0}(x)$ for each $x \in$ $X$. Now arrange the vertices of $X$ in order $x_{1}, x_{2}, \ldots, x_{|X|}$, such that if $i<j$ then $\varepsilon\left(x_{i}\right) \geq \varepsilon\left(x_{j}\right)$. Observe that there is an optimal assignment for which $x_{i}$ is assigned 1 for every $i \leq t$, and $x_{i}$ is assigned 0 for every $i>t$, for some $t \in\{0, \ldots,|X|\}$. (Consider an assignment for which $\varphi\left(x_{i}\right)=0$ and $\varphi\left(x_{j}\right)=1$, for $i<j$, and observe that switching the assignments of $x_{i}$ and $x_{j}$ will increase $\sum_{i=0}^{1} \sum_{x: \varphi(x)=i} w_{i}(x)$ by an amount of $\varepsilon\left(x_{i}\right)-\varepsilon\left(x_{j}\right)$ while $\sum_{\{x, y\} \in E\left(G_{S}\right)} \mid \varphi(x)-$ $\varphi(y) \mid$ stays the same.) So we only need to try $|X|+1$ different assignments to the vertices in $X$ in order to find the optimal colouring when $\varphi(r)=1$. Let $V_{1}$ be the value of this optimal assignment over $X \cup\{r\}$. By the same method we can find the optimal assignment when $r$ is assigned 0 , whose value we denote by $V_{0}$. Now remove the vertices in $X$ from $G_{S}$, and change $w_{i}(r)$ to $V_{i}$ for $i=0,1$.

Let us now analyse the run time of this procedure. If $\varepsilon(v)>|X|$ for a vertex $v \in X$, then $\varphi(v)$ must be 1 in an optimal assignment. Similarly, $\varphi(v)=0$ if $\varepsilon(v)<-|X|$. Hence, we only have to sort at most $|X|$ vertices according to their value $\{-|X|, \ldots,|X|\}$, which we can do in time $O(|X|)$ using counting sort.

The value of the first tested single assignment $\varphi$ can be computed in time $O\left(\left|E\left(G_{S}[X \cup\{r\}]\right)\right|\right.$. The next assignment $\varphi^{\prime}$ we want to test differs in only one vertex $v$ from the last assignment. Hence, the only differences between $\varphi$ and $\varphi^{\prime}$ are in $E(\{v\}, X \cup\{r\} \backslash\{v\})$. Therefore we can compute the value of $\varphi^{\prime}$ in time $O(|N(v)|)$. This way, we can check all $|X|+1$ assignments in time $O\left(\left|E\left(G_{S}[X]\right)\right|\right)$. Since each 
edge of $E\left(G_{S}\right)$ belongs to exactly one block of $G_{S}$, the entire procedure runs in time $O\left(\left|E\left(G_{S}\right)\right|\right)$.

We now give a proof for Theorem 1. Given a connected signed graph $G$ on $m$ edges, by Lemma 3 we find the set $S$ from Proposition 5 in time $O(\mathrm{~km}$ ) (the case that $k$ is not decreased can only take $O(m)$ total time). Guess one of the at most $2^{3 k}$ partitions on $S$ and solve the corresponding MAX-CUT EXTENSION problem with Lemma 4.

Proof of Theorem 1 Let $(G, k)$ be an instance of SIGNED MAX- CUT AEE. Compute the 2-connected components of $G$ and apply Lemma 3 to a leaf block $X$ of $G$ to obtain an instance $\left(G^{\prime}, k^{\prime}\right)$. Repeat this procedure exhaustively or until $k^{\prime} \leq 0$.

If Rule 5 was applied, the only remaining vertex of $X$ in $G^{\prime}$ is the cut vertex in $X$. Thus we do not need to recompute the 2-connected components of $G$ and we can use Lemma 3 immediately again. This way, all applications of Rule 5 take time $O(|E(G)|)$ in total. For every other rule, it holds $k^{\prime} \leq k-1$. This means that the other rules are applied at most $k$ times and thus the whole procedure runs in time $O(k \cdot|E(G)|)$.

Let $S$ be the set of marked vertices. If $k^{\prime} \leq 0$, then $(G, k)$ is a "yes"-instance. Otherwise, $|S| \leq 3 k$. We guess a 2-colouring $\varphi_{S}: S \rightarrow\{0,1\}$ for the vertices in $S$; there are $2^{|S|} \leq 2^{3 k}=8^{k}$ such 2-colourings. For $\varphi_{S}$, we solve MAX-CUT EXTENSION on the clique forest $G-S$, where we try to extend $\varphi_{S}$ to a maximum cut in $G$.

Formally, for an assignment $\varphi: S \rightarrow\{0,1\}$ let $S_{i}=\{v \in S \mid \varphi(v)=i\}$ for $i=0,1$. For a vertex $v \in V(G) \backslash S$, define the weight functions $w_{0}(v):=\mid N_{G}^{+}(v) \cap$ $S_{0}|+| N_{G}^{-}(v) \cap S_{1} \mid$ and $w_{1}(v):=\left|N_{G}^{+}(v) \cap S_{1}\right|+\left|N_{G}^{-}(v) \cap S_{0}\right|$. Then remove the vertices of $S$ from $G$. By Proposition 5, the resulting graph $G_{S}=G-S$ is a clique forest. Let $p$ be the number of edges within $G[S]$ that are satisfied by the restriction of $\varphi$ to $G[S]$. Then for any assignment to the vertices of $G_{S}$, the maximum number of satisfied edges in $G$ is exactly equal to

$$
p+\sum_{\{x, y\} \in E\left(G_{S}\right)}|\varphi(x)-\varphi(y)|+\sum_{i=0}^{1} \sum_{x: \varphi(x)=i} w_{i}(x),
$$

where $\varphi: V\left(G_{S}\right) \rightarrow\{0,1\}$ is the desired bipartition. Thus, $(G, k)$ is a "yes"instance if and only if the instance of MAX-CUT EXTENSION has optimal value at least $|E(G)| / 2+(|V(G)|-1+k) / 4-p$. We can test this in time $O(m)$ for every assignment $\varphi_{S}$ according to Lemma 4 .

\section{A Linear Vertex Kernel for Signed Max-Cut AEE}

In this section we will show how to obtain a kernel with $O(k)$ vertices and thus prove Theorem 2. Let $G^{0}$ be the original graph, let $S$ be the set of marked vertices during the exhaustive application of Rules $1-7$ on $G^{0}$, and let $G^{r}$ be the resulting graph after the exhaustive application of our kernelization Rules 8-9 (to be defined later) on $G^{0}$.

Let $C$ be a block in the clique forest $G-S$. Define

$$
C_{\text {int }}=\left\{v \in V(C) \mid N_{G-S}(v) \subseteq V(C)\right\}
$$


as the interior of $C$, and $C_{\text {ext }}=V(C) \backslash C_{\text {int }}$ as the exterior of $C$. The block $C$ is called special if $C_{\text {int }} \cap N_{G}(S)$ is non-empty. Let $\mathcal{B}$ be the set of blocks in $G^{r}-S$ and let $\mathcal{B}^{\star}$ be the set of special blocks in $G^{r}-S$. A $\Delta$-block is a non-special block $C$ on exactly three vertices for which $\left|C_{\text {ext }}\right| \leq 2$.

If there is a (unique by Proposition 5) remaining vertex $v$ left after the exhaustive application of Rules 1-7, then add an induced 2-path $(v, w, x)$ to $G^{0}$, i.e., define $G^{\prime}=\left(V\left(G^{0}\right) \cup\{w, x\}, E\left(G^{0}\right) \cup\{\{v, w\},\{w, x\}\}\right)$. Then $\left(G^{\prime}, k+2\right)$ is an instance of MAX-CUT AEE, that by Proposition 4 is equivalent to $(G, k)$ because the excess of an induced 2-path equals $2 / 4$. Therefore, we can assume that every vertex gets removed during the exhaustive application of the reduction rules because we can assume that Rule 6 removes the path $(v, w, x)$ in the last iteration. Furthermore, as Rule 5 can then not be applied last, we can assume that at least one of the vertices that are removed in the last iteration is contained in $S$.

We will now use two-way reduction rules to reduce the size of $G^{0}-S$ by shrinking or merging blocks that satisfy certain conditions. These rules are similar to the twoway reduction rules by Crowston et al. [7]. However, our two-way reduction rules have the property that connected components of $G-S$ cannot "fall apart", i.e., two blocks in $G^{r}-S$ are reachable from each other if and only if the corresponding blocks in $G^{0}-S$ are reachable from each other. We can then show that Rules 1-7 can behave "equivalently" on $G^{r}$ as on $G^{0}$ (Lemma 7), i.e., that the same set $S$ of vertices can also be marked in $G^{r}$. This is the crucial idea which allows us to obtain better kernelization results than previous work, as it allows the following analysis.

To show size bounds for our kernel $G^{r}$, we first argue that $\left(G^{r}, k\right)$ is a "yes"-instance if there are many special blocks. Intuitively, if there are many special blocks in $G^{r}-S$, we can find large pairwise vertex-disjoint stars $Y_{S}$ for every $s \in S$, whose leaves are internal vertices of blocks of $G^{r}-S$. The excess of such a star $Y_{s}$ grows linearly in its size because a star is a bipartite graph. We then (hypothetically) modify Rules 1-7 in such a way that whenever a vertex $s \in S$ is about to be removed, we additionally remove the associated star $Y_{S}$. We can distribute the internal vertices of blocks from $G^{r}-S$ in such a way to the different stars $Y_{S}$ that the generated intermediate graphs during the exhaustive application of these rules are all still connected. Therefore we can conclude with Proposition 2 that the excess of $G^{r}$ can only be by $O(|S|)$ smaller than the total excess of all the stars $Y_{s}$. Hence, we can show that there are only $O(k)$ special blocks or $\left(G^{r}, k\right)$ is a "yes"-instance (Lemma 10).

Next we limit the total number of blocks in $G^{r}-S$ by $O(k)$. On a high level, Rule 8 deletes two internal vertices of a block and Rule 9 merges two $\Delta$-blocks. There can only be $O(k)$ blocks in $G^{r}-S$ with an even number of vertices (Lemma 12) because every block corresponds to an application of Rules 1-7 where $k$ was decreased (every application of a rule can "generate" only one block of $G^{r}-S$ and the only case in which $k$ is not decreased is when Rule 5 removes an even number of vertices, which together with their anchor form a block of odd order).

On the other hand, non-special blocks of odd order can be shrinked by Rule 8. If they have only at most two external vertices, they eventually become $\Delta$-blocks. There cannot be more $\Delta$-blocks than non- $\Delta$-blocks (Lemma 11) because Rule 9 merges adjacent $\Delta$-blocks. We conclude in Lemma 13 that the total number of blocks is in $O(k)$. 
The total number of external vertices in blocks of $G^{r}-S$, i.e., the number of cut vertices, is of course bounded by the total number of blocks in $G^{r}-S$. Due to Rule 8, every non-special block in $G^{r}-S$ contains at most as many internal as external vertices. This is why the total number of vertices in non-special blocks is also bounded by $O(k)$. In order to bound the number of vertices in special blocks (Lemma 15), we reuse the approach of Lemma 10 . The difference is that we do not take only single vertices from special blocks in order to build stars $Y_{s}, s \in S$, but larger sets of internal vertices from each block. The idea will be described in more detail before Lemma 14 . This will complete the proof.

\subsection{Kernelization Rules}

We now give our two-way reduction rules, which on an input $(G, k)$ produce an instance $\left(G^{\prime}, k\right)$ of SIGNED MAX-CUT AEE. Note that the parameter $k$ does not change. We call a rule 2 -safe if $(G, k)$ is a "yes"-instance if and only if $\left(G^{\prime}, k\right)$ is. The first rule is again due to Crowston et al. [7], who showed it to be 2-safe; here we contribute its improved run time analysis. Recall our assumption that (without loss of generality) $G-S$ does not contain any positive edges.

Reduction Rule 8 Let $C$ be a block in $G-S$. If there exists $X \subseteq C_{\text {int }}$ such that $|X|>\frac{|V(C)|+\left|N_{G}(X) \cap S\right|}{2} \geq 1, N_{G}^{+}(x) \cap S=N_{G}^{+}(X) \cap S$ and $N_{G}^{-}(x) \cap S=N_{G}^{-}(X) \cap S$ for all $x \in X$, then delete two arbitrary vertices $x_{1}, x_{2} \in X$.

Reduction Rule 9 Let $C_{1}, C_{2}$ be $\Delta$-blocks in $G-S$ which share a common vertex $v$. Make a block out of $V\left(C_{1}\right) \cup V\left(C_{2}\right)$, i.e., add negative edges $\left\{\{u, w\} \mid u \in V\left(C_{1}\right) \backslash\right.$ $\left.\{v\}, w \in V\left(C_{2}\right) \backslash\{v\}\right\}$ to $G$.

The combination of these two rules is a powerful tool to eliminate non-special blocks of odd order: Rule 8 ensures that in every non-special block $C$ it holds $\left|C_{\text {int }}\right| \leq\left|C_{\text {ext }}\right|$ (otherwise, set $X$ to $C_{\text {int }}$, then $|X|>\frac{|V(C)|}{2}=\frac{\left|C_{\text {int }}\right|+\left|C_{\text {ext }}\right|}{2} \geq 1$, where the last inequality holds because every non-special block contains at least two vertices). This means that Rule 8 reduces non-special blocks $C$ of odd order with $\left|C_{\text {ext }}\right| \leq 2$ to blocks of order 1 (i.e., deleting the block if $C$ was a leaf block of odd order) or order 3 . In the latter case, $C$ becomes a $\Delta$-block.

Rule 9 combines two adjacent $\Delta$-blocks to a block of order 5 . If the common external vertex of the $\Delta$-blocks is not adjacent to $S$, the resulting block is also non-special and can therefore again be shrinked by Rule 8 . We can therefore contract arbitrarily large chains of non-special blocks.

Lemma 5 Rules 8-9 are 2-safe. If they are applied to a connected graph $G$, then the resulting graph $G^{\prime}$ is also connected.

Proof For Rule 8 we have nothing to show because it is Rule 8 from Crowston et al. [7]. Rule 9 does not destroy connectivity, as nothing is deleted. It remains to show that Rule 9 is 2-safe.

Let $C=V\left(C_{1}\right) \cup V\left(C_{2}\right)$. Consider a partition $V_{1} \uplus V_{2}$ of $V(G)$. This partition induces balanced subgraphs $H$ in $G$ and $H^{\prime}$ in $G^{\prime}$ (see Proposition 3). Let us first 
assume that neither $V\left(C_{1}\right)$ nor $V\left(C_{2}\right)$ is completely contained in either $V_{1}$ or $V_{2}$. Then also $\left|V_{1} \cap C\right| \leq 3$ and $\left|V_{2} \cap C\right| \leq 3$. Because $G[C]$ is the union of two triangles and $G^{\prime}[C]$ is a clique of size 5, the partition induces subgraphs $H[C]$ and $H^{\prime}[C]$ with exactly four and exactly six edges, respectively. Hence, $\left|E\left(H^{\prime}\right)\right|=|E(H)|+2$. It also holds that $\operatorname{pt}\left(G^{\prime}\right)=\operatorname{pt}(G)+2$, as $G^{\prime}$ is equal to $G$ with four additional edges. It remains to show that there always is a partition which induces maximum balanced subgraphs for $G$ and $G^{\prime}$ such that neither $V\left(C_{1}\right)$ nor $V\left(C_{2}\right)$ is completely contained in one of the sides of the partition.

Therefore, let us assume w.l.o.g. that $V\left(C_{1}\right)$ be completely contained in $V_{1}$. Let $b$ be an internal vertex of $C_{1}$. Because $b$ is in $G$ as well as in $G^{\prime}$ only adjacent to vertices in $C$, it holds $\left|N_{H}(b)\right|=0$ and $\left|N_{H^{\prime}}(b)\right| \leq 2$. As it also holds $\left|N_{G}(b)\right|=2$ and $\left|N_{G^{\prime}}(b)\right|=4$, the partition $\left(V_{1} \backslash\{b\}\right) \uplus\left(V_{2} \cup\{b\}\right)$ induces balanced subgraphs of $G$ and $G^{\prime}$ that cannot be smaller than $H$ and $H^{\prime}$, respectively. This completes the proof.

Lemma 6 Given $S$, Rules 8-9 can be applied exhaustively to $G^{0}$ in total time $O(m)$.

Proof First observe that we can compute the blocks of $G^{0}-S$ in time $O(m)$ using any linear-time algorithm for detecting 2-connected components. Then we can store for every cut vertex the list of $\Delta$-blocks it belongs to. An update of this list after an application of one of the rules can be done in constant time. As Rule 9 can be applied $O(n)$ times and merging two $\Delta$-blocks takes constant time, all applications of this rule can be done in total time $O(n)$.

We now discuss the run time of Rule 8 . Let $B$ be a block in $G^{0}-S$. Let $S_{B}$ be the vertices from $S$ adjacent to $B$, i.e., $S_{B}=S \cap N_{G^{0}}(B)$. Consider the auxiliary graph $H_{B}:=\left(S_{B} \cup B_{\text {int }}, E\left(S_{B}, B_{\text {int }}\right)\right)$. We use partition refinement to find the partition $V_{1} \uplus \ldots \uplus V_{p}=B_{\text {int }}$ of the internal vertices of $B$ such that two vertices $v, w$ are in the same set $V_{i}$ if and only if $N_{G^{0}}^{+}(v)=N_{G^{0}}^{+}(w)$ and $N_{G^{0}}^{-}(v)=N_{G^{0}}^{-}(w)$. To be more precise, let $\mathcal{P}$ be a partition of $B_{\text {int }}$. Initially, $\mathcal{P}=\left\{B_{\text {int }}\right\}$. Then for every $v \in V$, we refine $\mathcal{P}$ by $N(v)$, i.e., we split every set $X \in \mathcal{P}$ into three sets $X \cap N^{+}(v)$, $X \cap N^{-}(v)$, and $X \backslash N(v)$. Using appropriate data structures [25], this refinement can be executed in time $O(|N(v)|)$ in every iteration. Thus, we can compute $V_{1} \uplus \ldots \uplus V_{p}$ in time $O\left(\left|V\left(H_{B}\right)\right|+\left|E\left(H_{B}\right)\right|\right)$. As every edge of $G^{0}$ is in at most one auxiliary graph and every vertex $s \in S$ is in at most $\left|N_{G^{0}}(s)\right|$ auxiliary graphs, we can do these computations for all blocks of $G^{0}-S$ in total time $O(m)$.

For a block $B$, we can find the biggest class $V_{i *}$ in linear time. Then, as long as $B$ does not get merged due to Rule $9, V_{i^{*}}$ is the only class from which Rule 8 can delete vertices. (This is a bit subtle, as $\left|V_{i^{*}}\right|$ can be $\frac{|V(B)|-1}{2}$ after deleting vertices from $V_{i^{*}}$, but then $N_{G^{0}}\left(V_{i^{*}}\right) \cap S=\emptyset$; hence, every other $V_{i}$ has a neighbour in $S$ and would thus need size strictly larger than $\frac{|V(B)|+1}{2}$ in order to meet the requirements of Rule 8).

It is trivial to compute the number of possible applications of Rule 8 to $V_{i^{*}}$. This means that we can apply Rule 8 exhaustively (without allowing Rule 9 to be applied in the meantime) on $G^{0}-S$ in total time $O(m)$.

Now observe that every block newly created by Rule 9 has constant size. Hence, if we have also computed a partition according to the neighbourhoods for the whole graph $G^{0}-S$ (in time $O(m)$ ), we can check in constant time whether we can apply 
Rule 8 again on a newly created block. As this happens $O(n)$ times, the total time required for all applications of Rule 8 is $O(m)$.

Lemma 7 Rules 1-7 can be applied exhaustively to the graph $G^{r}$ in such a way that the set $S^{\prime}$ of marked vertices is equal to $S$. Moreover, if only the Rules $3 / 5 / 6 / 7$ are applied to $G^{0}$, the same set of rules is applied to $G^{r}$.

The last part of the lemma will be needed later in Sect. 5.2. It can easily be seen by the fact that the mentioned rules are the only rules applicable to an instance of MAX-CUT AEE (where all edges are negative).

Proof of Lemma 7 It suffices to show the following: Let $G$ be a connected graph and let $G^{\prime}$ be the resulting graph after a single application of Rule 8 or Rule 9 to $G$. Then Rules 1-7 can be applied exhaustively to $G^{\prime}$ in such a way that the same vertices are marked as during the exhaustive application of these rules to $G$.

Let $\left(G=G_{0}, G_{1}, \ldots, G_{q}\right.$ ) be the sequence of graphs generated by the exhaustive application of Rules $1-7$ to $G$ and let $S$ be the set of marked vertices. Let $X_{i}:=V\left(G_{i}\right) \backslash$ $V\left(G_{i+1}\right)$ for $i<q$ be the set of vertices removed in the $i$-th application of one of the rules (we start counting at 0 here for convenience).

- We first consider the case that $G^{\prime}$ resulted from an application of Rule 8 . Let $C, X, x_{1}, x_{2}$ be defined like in this rule. Furthermore, let $i$ and $j$ be the indices such that $x_{1} \in X_{i}$ and $x_{2} \in X_{j}$. W.l.o.g., $i \leq j$. Because $x_{1} \notin S$, the set $X_{i}$ is removed by one of the Rules $2 / 5 / 7$. The only possible neighbour of $x_{1}$ remaining in $G_{i+1}$ is either contained in $S$ or an external vertex in $G-S$. Because $x_{2}$ is by the definition of Rule 8 an internal vertex of $G-S$, it follows that $i=j$.

Consider now the sequence of graphs $\left(G^{\prime}=G_{0}^{\prime}, G_{1}^{\prime}, \ldots, G_{q}^{\prime}\right)$ defined by $G_{i^{\prime}}^{\prime}=$ $G_{i^{\prime}}-\left\{x_{1}, x_{2}\right\}$ for every index $i^{\prime}$. Note that $G_{i^{\prime}+1}^{\prime}=G_{i^{\prime}}^{\prime}-X_{i^{\prime}}$ for every index $i^{\prime} \neq i$, and $G_{i+1}^{\prime}=G_{i}^{\prime}-\left(X_{i} \backslash\left\{x_{1}, x_{2}\right\}\right)$. We show that the exhaustive application of Rules $1-7$ can yield this sequence of graphs.

Consider first the iteration $i^{\prime}=i$. Because $X_{i}$ contains vertices that are not in $S$, this set must be removed from $G_{i}$ by one of the Rules 2/5/7. If Rule 2 removes $X_{i}$ from $G_{i}$, then Rule 1 or Rule 2 can remove $X_{i} \backslash\left\{x_{1}, x_{2}\right\}$ from $G_{i}^{\prime}$, depending on whether $X_{i} \backslash\left\{x_{1}, x_{2}\right\} \subseteq S$. If Rule 5 removes $X_{i}$ from $G_{i}$, then either the same rule can remove $X_{i} \backslash\left\{x_{1}, x_{2}\right\}$ from $G_{i}^{\prime}$ or $G_{i}^{\prime}=G_{i+1}^{\prime}$ due to $\left|X_{i}\right|=2$. If Rule 7 removes $X_{i}$ from $G_{i}$, then $|X| \geq 3$ by the definition of Rule 8 and therefore the same rule can remove $X_{i} \backslash\left\{x_{1}, x_{2}\right\}$ from $G_{i}^{\prime}$.

For every other iteration we just have to ensure that connectivity is preserved, i.e., it suffices to show that for every $Y \subseteq V\left(G_{i^{\prime}}^{\prime}\right)$ and two vertices $a, b \in V\left(G_{i^{\prime}}^{\prime}\right) \backslash Y$, the vertices $a, b$ are in the same connected component of $G_{i^{\prime}}^{\prime}-Y$ if and only if they are in the same connected component of $G_{i^{\prime}}-Y$.

Let $P$ be a shortest $a$-b-path in $G_{i^{\prime}}-Y$ (if one exists). If $P$ does not contain $x_{1}$ and $x_{2}$, then $P$ also exists in $G_{i^{\prime}}^{\prime}$. Otherwise, $P$ contains exactly one of these two vertices because they share the same closed neighbourhood. Let w.l.o.g. $x_{1}$ be the vertex contained in $P$. Because $x_{1}$ is an internal vertex in $G-S$, the predecessor and the successor of $x_{1}$ in $P$ must be contained in $C_{\text {ext }} \cup S$. This means that $|V(C)|+\left|N_{G}(X) \cap S\right| \geq\left|\left\{x_{1}, x_{2}\right\}\right|+2 \geq 4$ and thus $|X| \geq 3$, i.e., 
there is a vertex $x_{3} \in V(G)$ with the same closed neighbourhood as $x_{1}$. With the same arguments as for $x_{2}$ one can conclude that also $x_{3} \in X_{i}$. Therefore, $x_{3}$ is contained in $G_{i^{\prime}}^{\prime}$ and thus we can replace $x_{1}$ by $x_{3}$ in $P$. Hence, $a$ and $b$ are in the same connected component of $G_{i^{\prime}}^{\prime}-Y$ if they are in the same connected component of $G_{i^{\prime}}$. The converse direction holds trivially.

- Now consider the case that $G^{\prime}$ resulted from an application of Rule 9. Let $v, C_{1}, C_{2}$ be defined like in this rule. Because $C_{1}$ and $C_{2}$ are both $\Delta$-blocks and hence not special, the following is well-defined: Let $i$ be the index such that $X_{i} \subseteq$ $V\left(C_{1}\right) \cup V\left(C_{2}\right)$ and $X_{i}$ is removed by Rule 5 using $v$ as anchor, and let $j$ be the index such that $v \in X_{j}$ and $X_{j}$ is removed by Rule 5 using some anchor $w$. Let $\ell$ be the index such that $w \in X_{\ell}$.

We define the sequence of graphs $\left(G^{\prime}=G_{0}^{\prime}, G_{1}^{\prime}, \ldots, G_{q}^{\prime}\right)$ in the following way: In iteration $i$, do nothing, i.e., $G_{i+1}^{\prime}=G_{i}^{\prime}$. In iteration $j$, remove $X_{i} \cup X_{j}$ from $G_{j}^{\prime}$. In all other iterations $i^{\prime}$, remove $X_{i^{\prime}}$ from $G_{i^{\prime}}^{\prime}$. We show again that this sequence can be generated by the exhaustive application of Rules 1-7 to $G^{\prime}$.

Consider first the $j$-th iteration. Obviously Rule 5 can remove $X_{i} \cup X_{j}$ from $G_{j}^{\prime}$ using $w$ as anchor. For every other iteration, observe that $w$ is the only vertex in $V(G) \backslash\left(X_{i} \cup X_{j}\right)$ with $N_{G}(w) \neq N_{G^{\prime}}(w)$. Hence, one can easily check for every single rule and every iteration $i^{\prime} \neq \ell$ that whenever a rule removes $X_{i^{\prime}}$ from $G_{i^{\prime}}$, it can also remove the same set from $G_{i^{\prime}}^{\prime}$ (intuitively because the rule cannot "see" the difference between $G_{i}^{\prime}$ and $G_{i}$ ). It remains to look at iteration $\ell$. Because $G_{i^{\prime}}^{\prime}=G_{i^{\prime}}$ for every index $i^{\prime}>j$, it follows $G_{\ell}^{\prime}=G_{\ell}$ and thus also the $\ell$-th iteration is safe. This completes the proof.

\subsection{Bounding the Kernel Size}

After having shown Lemma 7, we can now turn to the task of showing a linear kernel size. We first show some auxiliary lemmas, which will be useful in the proofs of the main Lemmas 10/15.

For the whole section, let $\left(G^{r}=G_{0}, \ldots, G_{q}\right)$ be the sequence of graphs generated by the exhaustive application of Rules 1-7 to $G^{r}$ such that the set of marked vertices is $S$, and let $X_{i}:=V\left(G_{i}\right) \backslash V\left(G_{i+1}\right)$ be the set of vertices removed in the $(i+1)$-th application. Recall that we assumed, without loss of generality, that $G_{q}$ is the empty graph, i.e., $\bigcup_{i<q} X_{i}$ covers $V\left(G^{r}\right)$.

Definition 3 Let Int be the set of internal vertices in $G^{r}-S$. Furthermore we call a vertex $v$ fixed if it is removed as the only vertex in an application of Rule 5 with anchor in $S$, but $v$ is not an isolated vertex in $G^{r}-S$. Denote by $F$ the set of all fixed vertices. Let Cand:=Int $\backslash F$ be the set of candidate vertices.

Fixed vertices play a special role in our clique forest. If a block $B$ contains a fixed vertex $v$, then $V(B) \backslash\{v\}$ is removed by Rule 5 using $v$ as anchor, before in a later iteration $\{v\}$ is removed by Rule 5 as the last vertex of its connected component of $G^{r}-S$. As a consequence, the number of blocks in $G^{r}-S$ does not increase when Rule 5 removes a fixed vertex. In other words, the total number of applications of Rules $2 / 5 / 7$ is equal to the number of blocks in $G^{r}-S$ minus $|F|$. It is also clear that 
there can only be at most $k$ fixed vertices or $\left(G^{r}, k\right)$ is a "yes"-instance. The name "fixed" stems from the fact that later in the proofs of Lemma 10 and Lemma 15 we do not want to "reattach" these fixed vertices.

Lemma 8 Let $G$ be a connected signed graph, and let $X$ be a vertex set from $G$ such that $G[X]$ is a clique containing only negative edges. If Rule 5 can remove $X$ using some anchor $w \in V(G)$, then $\operatorname{ex}(G) \geq \operatorname{ex}(G-X)+\min \left\{\left|X \cap N_{G}^{+}(w)\right|,\left|X \cap N_{G}^{-}(w)\right|\right\}$.

Proof Let $\alpha:=\min \left\{\left|X \cap N_{G}^{+}(w)\right|, \mid X \cap N_{G}^{-}(w)\right\}$. Order the vertices $\left\{x_{1}, \ldots, x_{\ell}\right\}=X$ in such a way that there is an index $i^{*}$ such that the edge $w x_{i}$ is positive if and only if $i \leq i^{*}$. Because $G[X]$ is a clique containing only negative edges, the partition $\left(\left\{x_{1}, \ldots, x_{\lfloor\ell / 2\rfloor}\right\},\left\{x_{\lfloor\ell / 2\rfloor+1}, \ldots, x_{\ell}\right\}\right)$ induces a balanced subgraph of size $\operatorname{pt}(G[X])+\operatorname{ex}(G[X])$. Now we add the vertex $w$ to the left-hand side.

If $i^{*} \leq\left\lfloor\frac{\ell}{2}\right\rfloor$, then $\alpha=i^{*}$ and $\left(\left\{w, x_{1}, \ldots, x_{\lfloor\ell / 2\rfloor}\right\},\left\{x_{\lfloor\ell / 2\rfloor+1}, \ldots, x_{\ell}\right\}\right)$ induces a balanced subgraph of size

$$
\operatorname{pt}(G[X])+\operatorname{ex}(G[X])+i^{*}+(\ell-\lfloor\ell / 2\rfloor)=\operatorname{pt}(G[X])+\operatorname{ex}(G[X])+\lceil\ell / 2\rceil+\alpha .
$$

If $i^{*} \geq\left\lceil\frac{\ell}{2}\right\rceil$, then $\alpha=\ell-i^{*}$ and $\left(\left\{w, x_{1}, \ldots, x_{\lceil\ell / 2\rceil}\right\},\left\{x_{\lceil\ell / 2\rceil+1}, \ldots, x_{\ell}\right\}\right.$ induces a balanced subgraph of size

$$
\operatorname{pt}(G[X])+\operatorname{ex}(G[X])+\lceil\ell / 2\rceil+\left(\ell-i^{*}\right)=\operatorname{pt}(G[X])+\operatorname{ex}(G[X])+\lceil\ell / 2\rceil+\alpha .
$$

Because $G[X \cup\{w\}]$ contains $\ell$ edges and one vertex more than $G[X]$, it follows that $\operatorname{pt}(G[X \cup\{w\}])=\operatorname{pt}(G[X])+\ell / 2+1 / 4$ and thus

$$
\operatorname{ex}(G[X \cup\{w\}]) \geq \operatorname{ex}(G[X])+\lceil\ell / 2\rceil+\alpha-\ell / 2-1 / 4 \geq \alpha,
$$

where the last inequality follows from the fact that $\operatorname{ex}(G[X])=\frac{1}{4}$ if $\ell$ is even. The lemma now follows from Proposition 4.

Lemma 9 Let $\left(G, k^{\prime}\right)$ be an instance of SIGNED MAX-CUT AEE that arises during the exhaustive application of Rules $1-7$ to $\left(G^{r}, k\right)$. If in the next step Rule 5 removes a vertex set $X$ from $G$ with an anchor $s \in S$, and if the connected component of $G^{r}-S$ that contains $X$ consists of a single block, then $\operatorname{ex}(G) \geq \operatorname{ex}(G-X)+\frac{1}{4}$.

Proof Let $C$ be the block of $G^{r}-S$ containing $X$. For a better understanding, we first point out the relation between $C$ and $X$ : Either $V(C)=X$ or the single vertex in $X$ was the anchor of $V(C) \backslash X$ in a previous iteration and now this single vertex is removed by Rule 5 .

We now turn to the proof. If $|X|$ is odd, then the lemma follows immediately from Proposition 5. Otherwise $|X|$ is even, $X=V(C)$ and $N_{G}(x) \cap S=\{s\}$ for every $x \in X$. If $N_{G}^{+}(s) \cap X=\emptyset$ or $N_{G}^{-}(s) \cap X=\emptyset$, then Rule 8 would have eliminated $X$, as $X$ is an isolated block in $G^{r}-S$, i.e., it contains only internal vertices, which have all the same neighbourhood Hence, $\min \left\{\left|X \cap N_{G}^{+}(w)\right|, \mid X \cap N_{G}^{-}(w)\right\} \geq 1$. Because $G^{r}-S$ contains only negative edges, in particular $G[X]$ contains only negative edges. Therefore we can use Lemma 8. 
Fig. 1 An example where putting the fixed vertex $x_{1}$ into $Y_{s_{3}}$ disconnects the graph

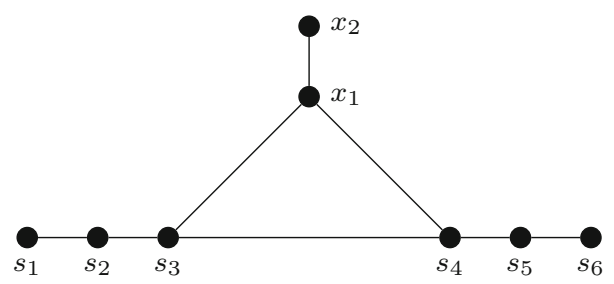

The following lemma is dedicated to bounding the number of special blocks to $O(k)$. Our approach is the following. Let $G$ be a connected vertex-induced subgraph of $G^{r}$ and let $s \in V(G) \cap S$. Furthermore, let $Y_{s}$ be a subgraph of $G$ that contains $s$ and internal vertices of blocks from $G^{r}-S$ that are all adjacent to $s$. If all these vertices are from different blocks, then $Y_{S}$ is a star, that is, it is a bipartite graph. Hence, if $G-Y_{S}$ is connected, it holds $\operatorname{ex}(G) \geq \operatorname{ex}\left(G-V\left(Y_{S}\right)\right)+\operatorname{ex}\left(Y_{S}\right)-\frac{1}{4}=\operatorname{ex}\left(G-V\left(Y_{S}\right)\right)+$ $\left(\left|E\left(Y_{S}\right)\right|-1\right) / 4$.

The idea is now to apply Rules $1-7$ again to $G^{r}$ in a modified way: Whenever a vertex from $S$ is removed, then at the same time also the star $Y_{S}$ is removed, resulting in a decrease of $k$ by roughly $\left|E\left(Y_{S}\right)\right|$. If there are more than $\Theta(k)$ many special blocks, i.e., blocks with internal vertices that are adjacent to $S$, then we should be able to identify $G^{r}$ as a "yes"-instance.

In order to make this approach work, we make sure that the following properties hold:

- All resulting graphs should be connected. In particular, we do not want to add fixed vertices to $Y_{s}$. For an illustration of the arising problem, take a look at the graph $G$ depicted in Fig. 1, in which Rule 6 can remove the sets $X_{1}=\left\{s_{1}, s_{2}, s_{3}\right\}$ and $X_{4}=\left\{s_{4}, s_{5}, s_{6}\right\}$ from the graph, whereas Rule 5 removes $X_{2}=\left\{x_{2}\right\}$ using $x_{1}$ as anchor, and $X_{3}=\left\{x_{1}\right\}$ using $s_{4}$ as anchor. By definition the vertex $x_{1}$ is a fixed vertex. If we added $x_{1}$ to $Y_{s_{3}}$, then $G_{1}^{\prime}=G\left[\left\{x_{2}, s_{4}, s_{5}, s_{6}\right\}\right]$ would not be connected.

- Whenever a set $X$ gets removed by Rule 5 during the "original application" and we want to remove the set $X^{\prime} \subseteq X$ in our modified setting, then $X^{\prime}$ should also be removable by Rule 5. This means that, if $s \in S$ is the anchor of $X$, then $Y_{s}$ cannot contain a vertex $w \in X$, as otherwise both $s$ and $w$ would be in the neighbourhood of $X^{\prime}$, contradicting the conditions of Rule 5 .

Lemma 10 If $G^{r}-S$ has more than $11 k$ special blocks, then $\left(G^{r}, k\right)$ is a "yes"instance of SIGNED MAX- CUT AEE.

Proof For a vertex $s \in S$, let $W_{s}$ be the union of all vertex sets $X_{i}$ such that $s$ is the anchor when Rule 5 removes $X_{i}$ from $G_{i}$. Furthermore, let $\Gamma:=\emptyset$ be the set of reattached vertices.

For every $i=q-1, \ldots, 0$ in decreasing order, do the following procedure successively for every $s \in X_{i} \cap S$ : Let $Y_{s} \subseteq G^{r}\left[\left(\right.\right.$ Cand $\left.\left.\backslash W_{s}\right) \cup\{s\}\right]$ be a maximum vertex-induced star centred in $s$. Add $V\left(Y_{S}\right) \backslash\{s\}$ to $\Gamma$.

We define a sequence of graphs $\left(G^{r}=G_{0}^{\prime}, \ldots, G_{q}^{\prime}\right)$ in the following way: For every $i \in\{0, \ldots, q-1\}$, let $X_{i}^{\prime}:=\left(X_{i} \backslash \Gamma\right) \cup \bigcup_{s \in X_{i} \cap S} V\left(Y_{s}\right)$, and let $G_{i+1}^{\prime}:=G_{i}^{\prime}-X_{i}^{\prime}$. 
Claim 1 For every $i=0, \ldots, q-1$, the following properties hold.

1. The graph $G_{i}^{\prime}$ is a vertex-induced subgraph of $G_{i}$ with $G_{i}-$ Cand $=G_{i}^{\prime}-$ Cand.

2. Both $G^{r}\left[X_{i}^{\prime}\right]$ and $G_{i}^{\prime}$ are connected.

Proof of the claim Let $s \in S$ and $w \in V\left(Y_{S}\right) \backslash\{s\}$. As $w$ is not contained in $S$, it originally got removed by Rule 2/5/7 and this happened not before $s$ got removed (by the definitions of these rules). Hence, every vertex of $\Gamma$ cannot be removed later than originally, which is why $G_{i}^{\prime}$ must be fully contained in $G_{i}$. Furthermore, as only candidate vertices of $G^{r}-S$ get reattached, the claim $G_{i}-$ Cand $=G_{i}^{\prime}-$ Cand follows trivially.

For the second part, first note that the subgraphs $G^{r}\left[X_{i}^{\prime} \cap S\right]$ and $Y_{s}$ for all $s \in X_{i} \cap S$ are connected. If Rule 5 removes $X_{i}$ from $G_{i}$, then $X_{i} \cap S=\emptyset$, i.e., $G^{r}\left[X_{i}^{\prime}\right]$ is a vertexinduced subgraph of the clique $G^{r}\left[X_{i}\right]$ and thus connected. If Rule 2/7 removes $X_{i}$ from $G_{i}$, then $N_{G_{i}}\left(X_{i} \backslash S\right) \subseteq X_{i} \cap S$, i.e., each vertex $s^{\prime} \in S \cap X_{j}$ for $j>i$ is non-adjacent to $X_{i} \backslash S$ in $G^{r}$. Hence, at least one star $Y_{s}, s \in X_{i} \cap S$, contains a vertex $v$ from $X_{i} \backslash S$. The graph $G^{r}\left[\left(X_{i}^{\prime} \cap X_{i}\right) \backslash S\right]$ is a vertex-induced subgraph of the clique $G^{r}\left[X_{i} \backslash S\right]$ and thus connected. Hence, every vertex of $X_{i}^{\prime}$ is adjacent to $X_{i} \cap S=X_{i}^{\prime} \cap S$ or to $v$. Thus, $G^{r}\left[X_{i}^{\prime}\right]$ is connected.

Regarding $G_{i}^{\prime}$, we show that for every $v, v^{\prime} \in V\left(G_{i}^{\prime}\right)$ there is a $\left[v, v^{\prime}\right]$-path in $G_{i}^{\prime}$. The vertices $v$ and $v^{\prime}$ are also contained in $G_{i}$ because $G_{i}^{\prime}$ is a subgraph of $G_{i}$. Because $G_{i}$ is connected, there is a path $v=p_{1}, \ldots, p_{\ell}=v^{\prime}$ in $G_{i}$. If this path is not fully contained in $G_{i}^{\prime}$, let $j, j^{\prime} \in\{1, \ldots, \ell\}$ be indices such that $p_{j}, p_{j^{\prime}} \in V\left(G_{i}^{\prime}\right)$ and $p_{j+1}, \ldots, p_{j^{\prime}-1} \notin V\left(G_{i}^{\prime}\right)$. We show that there are vertices $w, w^{\prime} \in V\left(G_{i}^{\prime}\right)$ for which $G^{r}\left[\left\{p_{j}, w, w^{\prime}, p_{j^{\prime}}\right\}\right]$ is connected. This suffices to show the claim, as $G_{i}^{\prime}$ is a vertex-induced subgraph of $G^{r}$.

Because $G_{i}-$ Cand $=G_{i}^{\prime}$ - Cand, the vertices $p_{j+1}, \ldots, p_{j^{\prime}-1}$ must all be nonfixed internal vertices, and because they form a path, they must all belong to the same block $C$ of $G^{r}-S$. There are now two possibilities for $p_{j}$ and $p_{j^{\prime}}$ : Each of the two is either an external vertex of $C$ or contained in $S$. If $p_{j}$ is adjacent to every internal vertex of $C$, we can set $w:=p_{j}$.

Otherwise $p_{j} \in S$. Let $d$ be the index such that $p_{j} \in X_{d}$. Because $p_{j} \in V\left(G_{i}\right)$, it holds $d \geq i$. Furthermore, as $p_{j+1} \notin V\left(G_{i}^{\prime}\right)$, it holds $p_{j+1} \in X_{t}^{\prime}$ for some $t<i \leq d$. This means that $Y_{p_{j}}$ contains a vertex $w$ from $C_{\text {int }}$, for otherwise $p_{j+1}$ could have been added to $Y_{p_{j}}$. In the same way we can find a vertex $w^{\prime} \in\left(C_{\text {int }} \cap Y_{p_{j^{\prime}}}\right) \cup\left\{p_{j^{\prime}}\right\}$. In any case, $w, w^{\prime} \in V(C) \cap V\left(G_{i}^{\prime}\right)$, and $\left\{w, w^{\prime}\right\} \in E\left(G_{i}^{\prime}\right)$. This shows the claim. $\diamond$

Claim 2 If $|\Gamma| \geq 5 k$, then $\left(G^{r}, k\right)$ is a “yes"-instance.

Proof of the claim Let $i \in\{0, \ldots, q-1\}$. If $X_{i}^{\prime}=\emptyset$, then trivially $\operatorname{ex}\left(G_{i+1}^{\prime}\right)=$ $\operatorname{ex}\left(G_{i}^{\prime}\right)$. Therefore we assume $X_{i}^{\prime} \neq \varnothing$ from now on.

If $X_{i}$ is removed by Rule 5, then $X_{i} \cap S=\emptyset$, i.e., $X_{i}^{\prime} \subseteq X_{i}$ and thus $N_{G_{i}^{\prime}}\left(X_{i}^{\prime}\right) \subseteq$ $N_{G_{i}}\left(X_{i}\right)=\{v\}$ for some anchor $v$. Because an anchor cannot be a candidate vertex by definition, and because $G_{i}^{\prime}-$ Cand $=G_{i}-$ Cand, the vertex $v$ is also contained in $G_{i}^{\prime}$. This means that Rule 5 can remove $X_{i}^{\prime}$ from $G_{i}^{\prime}$ with the same anchor $v$. Thus, $\operatorname{ex}\left(G_{i}^{\prime}\right) \geq \operatorname{ex}\left(G_{i+1}^{\prime}\right)$.

Consider now the case that a rule different to Rule 5 removes $X_{i}$ from $G_{i}$. Then $X_{i}^{\prime} \cap S \neq \emptyset$. For every vertex $s \in X_{i}^{\prime} \cap S$, the subgraph $Y_{s}$ is a star, and thus by 
Proposition 4 it holds $\operatorname{ex}\left(Y_{S}\right)=\frac{\left|E\left(Y_{s}\right)\right|}{4}$. Furthermore, if $X^{\prime \prime}:=X_{i}^{\prime} \backslash \bigcup_{s \in X_{i}^{\prime} \cap S} V\left(Y_{S}\right)$ is non-empty, then $X^{\prime \prime}$ is a clique (removed by Rule 2/5/7) and thus connected.

Hence, repeated applications of Proposition 2 in appropriate order (such that all generated intermediate graphs are connected) then yields

$$
\operatorname{ex}\left(G_{i}^{\prime}\left[X_{i}^{\prime}\right]\right) \geq \sum_{s \in X_{i}^{\prime} \cap S} \frac{\left|E\left(Y_{s}\right)\right|}{4}-\frac{\left|X_{i}^{\prime} \cap S\right|}{4}=\sum_{s \in X_{i}^{\prime} \cap S} \frac{\left|E\left(Y_{S}\right)\right|-1}{4} .
$$

A further application results in

$$
\operatorname{ex}\left(G_{i}^{\prime}\right) \geq \operatorname{ex}\left(G_{i+1}^{\prime}\right)+\operatorname{ex}\left(G_{i}^{\prime}\left[X_{i}^{\prime}\right]\right)-\frac{1}{4}=\operatorname{ex}\left(G_{i+1}^{\prime}\right)+\sum_{s \in X_{i}^{\prime} \cap S} \frac{\left|E\left(Y_{S}\right)\right|-1}{4}-\frac{1}{4}
$$

It holds $|\Gamma|=\sum_{s \in S}\left|V\left(Y_{S}\right) \backslash\{s\}\right|=\sum_{s \in S}\left|E\left(Y_{S}\right)\right|$. Because $|S| \leq 3 k$ and only at most $k$ times a rule different to Rule 5 is applied (otherwise, $\left(G^{r}, k\right)$ would be a "yes"-instance), it follows that

$$
\operatorname{ex}\left(G_{0}^{\prime}\right) \geq \operatorname{ex}\left(G_{q}^{\prime}\right)+\sum_{s \in S} \frac{\left|E\left(Y_{s}\right)\right|-1}{4}-\frac{k}{4} \geq \frac{|\Gamma|-3 k-k}{4} \geq \frac{|\Gamma|-4 k}{4}
$$

which is larger than $\frac{k}{4}$ if $|\Gamma| \geq 5 k$. Therefore, $\left(G^{r}, k\right)$ is a "yes"-instance if $|\Gamma| \geq 5 k$. This shows the claim.

Up to now we have already shown that at most $5 k$ special blocks contribute a vertex to $\Gamma$ or $\left(G^{r}, k\right)$ is a "yes"-instance. It remains to find a bound for the number of special blocks $C$ that do not share a vertex with $\Gamma$.

Let $C$ be such a special block, and let $i$ be the largest index such that $X_{i} \cap V(C) \neq \varnothing$. If $C$ contains a fixed vertex, then $X_{i}$ consists of this fixed vertex and $k$ was decreased by 1 when Rule 5 removed $X_{i}$. If the intersection $N_{G^{r}}\left(C_{\text {Int }}\right) \cap S$ would contain a vertex $s^{\prime}$ that is not the anchor of $X_{i}$, then the star $Y_{s^{\prime}}$ could be enhanced by a vertex from $C_{\text {int }}$. Hence, $N_{G^{r}}\left(C_{\text {Int }}\right) \cap S$ consists of a single vertex $s$, which is the anchor of $X_{i}$. In particular, $X_{i}$ got removed by Rule 5 .

Let $Z$ be the connected component of $G^{r}-S$ containing the block $C$. We now consider the following two cases:

1. If $Z$ contains another special block $C^{\prime}$, then one vertex of $C^{\prime}$ is contained in $\Gamma$. This is because in every connected component of $G^{r}-S$ only at most one block can have an anchor in $S$ (namely, the one that is removed last) and only these blocks can contain fixed vertices. Hence, the number of such blocks $C$ is bounded by $|\Gamma|$.

2. Now let $Z$ not contain another special block. If $\left|X_{i}\right|$ is odd, then $k$ is decreased by 1 during the application of Rule 5 . If $C$ is an isolated block in $G^{r}-S$, then Lemma 9 assures that $\operatorname{ex}\left(G_{i}^{\prime}\right) \geq \operatorname{ex}\left(G_{i+1}^{\prime}\right)+\frac{1}{4}$.

It remains the case that $C$ is not an isolated block, but all other blocks of $Z$ are not special. Let $C^{\prime} \neq C$ be a leaf block of $Z$. As $C^{\prime}$ has not been eliminated 
by Rule 8 , it must contain exactly two vertices, namely an internal vertex and an external vertex $w$. Then $V\left(C^{\prime}\right) \backslash\{w\}$ got removed by Rule 5 using $w$ as anchor. Because $\left|V\left(C^{\prime}\right) \backslash\{w\}\right|=1$ is odd, $k$ was decreased by 1 in that iteration.

Combining these two observations yields that the case that $Z$ does not contain another special block can only occur at most $k$ times or $\left(G^{r}, k\right)$ is a "yes"-instance.

Hence, the number of special blocks that do not share a vertex with $\Gamma$ is bounded from above by $|\Gamma|+k$. This means that in total there are at most $2|\Gamma|+k$ special blocks. As we already pointed out that $\left(G^{r}, k\right)$ is a "yes"-instance if $|\Gamma| \geq 5 k$, the lemma follows.

Now that we have bounded the number of special blocks in $G^{r}-S$, we can turn to the task of bounding the total number of blocks in $G^{r}-S$. In the following lemmas we show that a constant fraction of all blocks is special.

Lemma 11 The number of $\Delta$-blocks in $G^{r}$ is at most the number of non- $\Delta$-blocks in $G^{r}$.

Proof Assume the contrary. By Rule 8, any leaf block $C$ of $G^{r}-S$ cannot be a $\Delta$ block, as otherwise one could set $X=C_{\text {int }}$ with $|X|=2>\frac{3}{2}=\frac{|V(C)|+\left|N_{G} r(X) \cap S\right|}{2}$. Hence, there are two $\Delta$-blocks $C_{1}, C_{2}$ which share a common vertex $v$. Then Rule 9 applies.

Definition 4 We define a blockforest $F$ of $G^{r}-S$ in the following way. For a connected component $Z$ of $G^{r}-S$, let $C_{R}$ be an arbitrary block in $Z$. For every block $C$ in $Z$, there is a vertex $v_{C}$ in $F$. Add an edge $\left\{v_{C_{R}}, v_{C}\right\}$ for every block $C$ sharing a vertex with $C_{R}$. Additionally, add an edge $\left\{v_{C_{1}}, v_{C_{2}}\right\}$ if $C_{1}$ and $C_{2}$ share a vertex and every path from a vertex in $C_{R}$ to a vertex in $C_{2}$ contains at least two vertices from $C_{1}$.

It is easy to verify that any block forest is actually a forest.

Lemma 12 If more than $k$ non-special blocks in $G^{r}-S$ have an even number of vertices, then $\left(G^{r}, k\right)$ is a "yes"-instance of SIGNED MAX-CUT AEE.

Proof Let $B$ be a non-special block of $G^{r}-S$, and let $w$ be the external vertex of $B$. Then Rule 5 removed $V(B) \backslash\{w\}$ using $w$ as anchor. If $|V(B)|$ is even, then $|V(B) \backslash\{w\}|$ is odd and hence $k$ was decreased by 1 in that iteration.

Lemma 13 If $G^{r}-S$ has more than $48 k$ blocks, then $\left(G^{r}, k\right)$ is a "yes"-instance of SIGNED MAX-CUT AEE. Otherwise, $G^{r}-S$ has at most $48 k$ external vertices, and $\sum_{B \in \mathcal{B}}\left|B_{\text {ext }}\right| \leq 96 k$

Proof Consider a leaf block $C$ of $G^{r}-S$ that is not special. Then $C$ cannot have at least three vertices due to Rule 8 . Every block with exactly one vertex must be special. Hence, every leaf block is either special or it has exactly two vertices, i.e., due to Lemma 10 and Lemma 12 there are at most $12 k$ blocks that are leaf blocks or have an even number of vertices.

Let $F$ be a block forest of $G^{r}-S$. Every leaf of $F$ corresponds to a leaf block in $G^{r}-S$ and every block $C$ in $G^{r}-S$ with $\left|C_{\text {ext }}\right| \geq 3$ corresponds to a vertex with 
degree at least three in $F$. Because in every forest the number of leaves is at least the number of vertices with degree at least three, there are at most $12 k$ such blocks.

Now consider one of the remaining blocks $C$. Then $C$ is not special, contains at most two external vertices, and $|V(C)|$ is odd. Because it cannot be shrunk by Rule 8 , it holds that $|V(C)|=3$ and thus $C$ is a $\Delta$-block. This means that with the above arguments we bounded the number of blocks that are not $\Delta$-blocks by $24 k$. Lemma 11 yields that there can only be up to $24 k \Delta$-blocks. From this the bound of $48 k$ blocks follows.

Let $U$ be the set of external vertices in $G^{r}-S$. Every external vertex in $G^{r}-S$ which is in $c(v)$ blocks induces $c-1 \geq 1$ edges in $F$. Because $F$ is a forest, it holds $|E(F)|<|V(F)| \leq 48 k$. Thus, $\sum_{B \in \mathcal{B}}\left|B_{\text {ext }}\right| \leq \sum_{v \in U} c(v) \leq|E(F)|+|U| \leq 96 k$.

Now we have bounds for the total number of blocks and the number of external vertices in $G^{r}-S$. The number of internal vertices of non-special blocks is easily upper-bounded by the number of external vertices (otherwise apply Rule 8). So the remaining challenge is to find an upper bound for the number of internal vertices in special blocks.

We use the same approach as for Lemma 10, which already bounded the number of special blocks. There we generated a vertex-induced star $Y_{S}$ for every $s \in S$, which contained internal vertices of $G^{r}-S$ that were all adjacent to $s$. Intuitively speaking, every special block adjacent to $s$ contributed a leaf to $Y_{s}$, which lead to a constant gain in our bounds for the excess of $G^{r}$. Now a constant gain per special block is not enough for our purposes. Instead we need a gain that grows proportionally to the number of internal vertices in a block.

Let $B$ be a special block of $G^{r}-S$. First note that due to Rule 8 at most $\left|B_{\text {ext }}\right|+$ $\left|B_{\text {int }}\right| / 2$ vertices of $B_{\text {int }}$ can be non-adjacent to $S$, i.e., it suffices to find a bound for $\left|N_{G^{r}}(S) \cap B_{\text {int }}\right|$. Let $s \in S$ be a vertex that is adjacent to $B_{\text {int }}$. Select subsets $U^{+} \subseteq N_{G^{r}}^{+}(s) \cap V\left(B_{\text {int }}\right), U^{-} \subseteq N_{G^{r}}^{-}(s) \cap V\left(B_{\text {int }}\right)$, and $\bar{U} \subseteq V\left(B_{\text {int }}\right) \backslash N_{G^{r}}(s)$ with the following properties:

- $\left|U^{+} \cup U^{-}\right|$is maximal.

-||$U^{+}|-| U^{-}||=|\bar{U}|+1$.

We will show that we can cover a constant fraction of all internal vertices of $B_{\text {int }}$ if we repeat this procedure for every vertex in $S$. Then we follow the lines of the proof of Lemma 8: We can subdivide $\bar{U}$ into to sets $\bar{U}^{+}$and $\bar{U}^{-}$such that $\left|U^{+} \cup \bar{U}^{+}\right|=$ $\left|U^{-} \cup \bar{U}^{-}\right|-1$. This means that $\left(U^{+} \cup \bar{U}^{+}, U^{-} \cup \bar{U}^{-}\right)$induces a maximum balanced subgraph of $G^{r}\left[U^{+} \cup U^{-} \cup \bar{U}\right]$. Then we add the vertex $s$ to the left-hand side of the partition and increase thereby the number of edges in the induced subgraph by $\left|U^{+} \cup U^{-}\right|$, whereas the Poljak-Turzík bound only increases by roughly the half of it. Thus the excess bound grows linearly in $\left|U^{+} \cup U^{-}\right|$and thus in $\left|N_{G^{r}}(s) \cap B_{\text {int }}\right|$.

Lemma 14 Let $G$ be a connected signed graph with a cut vertex $s \in V(G)$ such that for every connected component $C$ of $G-s$ the following properties hold:

- $C$ is a clique containing only negative edges.

- ||$N_{G}^{+}(s) \cap V(C)|-| N_{G}^{-}(s) \cap V(C)||=\left|V(C) \backslash N_{G}(s)\right|+1$. 
Then $\operatorname{ex}(G) \geq \frac{|V(G) \backslash\{s\}|}{4}$.

Proof Let $C$ be a connected component of $G-s$. Furthermore, let $U^{+}=V(C) \cap$ $N_{G}^{+}(s), U^{-}=V(C) \cap N_{G}^{-}(s)$, and $\bar{U}=V(C) \backslash N_{G}(s)$.

First note that $C$ is an odd clique, i.e., $\operatorname{ex}(C)=0$. Because ||$U^{+}|-| U^{-}||=|\bar{U}|+1$ and $G-s$ only contains negative edges, there is a partition $\bar{U}^{+} \uplus \bar{U}^{-}$of $\bar{U}$ such that $\left|U^{+} \cup \bar{U}^{+}\right|=\left|U^{-} \cup \bar{U}^{-}\right|-1$, i.e., $\left(U^{+} \cup \bar{U}^{+}, U^{-} \cup \bar{U}^{-}\right)$induces a balanced subgraph of size $\operatorname{pt}(C)$ in $C$. Then $\left(\{s\} \cup U^{+} \cup \bar{U}^{+}, U^{-} \cup \bar{U}^{-}\right)$induces a balanced subgraph of size $\operatorname{pt}(C)+\left|N_{G}(s) \cap V(C)\right|$, whereas $\operatorname{pt}(G[V(C) \cup\{s\}])=\operatorname{pt}(C)+$ $\frac{\left|N_{G}(s) \cap V(C)\right|}{2}+\frac{1}{4}$. Therefore,

$$
\operatorname{ex}(G[V(C) \cup\{s\}]) \geq \frac{\left|N_{G}(s) \cap V(C)\right|}{2}-\frac{1}{4}=\frac{|V(C)|}{4},
$$

where the last equality holds because $\left|V(C) \cap N_{G}(s)\right|=\left|V(C) \backslash N_{G}(s)\right|+1$. By Proposition 4, we conclude that $\operatorname{ex}(G) \geq \frac{|V(G) \backslash\{s\}|}{4}$.

Lemma 15 If there are more than $111 k$ internal vertices in special blocks in $G^{r}-S$, then $\left(G^{r}, k\right)$ is a "yes"-instance of SIGNED MAX-CUT AEE.

Proof For notational simplicity, all neighbourhoods in this proof are with respect to $G^{r}$.

For a vertex $s \in S$, let $W_{s}$ be the union of all vertex sets $X_{i}$ such that $s$ is the anchor when Rule 5 removes $X_{i}$ from $G_{i}$. Let $W:=\bigcup_{s \in S} W_{s}$. Furthermore, let $\Gamma:=\varnothing$ be the set of reattached vertices, and let $Y_{s}:=\{s\}$ for every $s \in S$. (Note that $Y_{s}$ is a set as opposed to a subgraph in the proof of Lemma 10, but this is merely a technical issue.)

For all blocks $B$ of $G^{r}-S$ and every $i=q-1, \ldots, 0$ in decreasing order, run the following procedure successively for every $s \in X_{i} \cap S$ : Let $X:=B_{\text {int }} \backslash\left(F \cup \Gamma \cup W_{s}\right)$. Let $U^{-} \subseteq X \cap N^{-}(s), U^{+} \subseteq X \cap N^{+}(v)$ and $\bar{U} \subseteq X \backslash N(s)$ with $\left|U^{-} \cup U^{+}\right|$ maximal such that ||$U^{-}|-| U^{+}||=|\bar{U}|+1$. If such sets exist, i.e., if $X \cap N(s) \neq \emptyset$, then add $U^{-} \cup U^{+} \cup \bar{U}$ to $Y_{s}$ and to $\Gamma$.

We define a sequence of graphs $\left(G^{r}=G_{0}^{\prime}, \ldots, G_{q}^{\prime}\right)$ in the following way: For every $i \in\{0, \ldots, q-1\}$, let $X_{i}^{\prime}:=\left(X_{i} \backslash \Gamma\right) \cup \bigcup_{s \in X_{i} \cap S} Y_{s}$, and let $G_{i+1}^{\prime}:=G_{i}^{\prime}-X_{i}^{\prime}$.

Claim 3 For every $i=0, \ldots, q-1$, the following properties hold.

1. The graph $G_{i}^{\prime}$ is a vertex-induced subgraph of $G_{i}$ with $G_{i}-$ Cand $=G_{i}^{\prime}-$ Cand.

2. Both $G^{r}\left[X_{i}^{\prime}\right]$ and $G_{i}^{\prime}$ are connected.

Proof of the claim The proof is identical to the proof of the corresponding claim in Lemma 10. For completeness we repeat it here.

Let $s \in S$ and $w \in V\left(Y_{S}\right) \backslash\{s\}$. As $w$ is not contained in $S$, it originally got removed by Rule 2/5/7 and this happened not before $s$ got removed (by the definitions of these rules). Hence, every vertex of $\Gamma$ cannot be removed later than originally, which is why $G_{i}^{\prime}$ must be fully contained in $G_{i}$. Furthermore, as only candidate vertices of $G^{r}-S$ get reattached, the claim $G_{i}-$ Cand $=G_{i}^{\prime}-$ Cand follows trivially.

For the second part, first note that the subgraphs $G^{r}\left[X_{i}^{\prime} \cap S\right]$ and $G\left[Y_{s}\right]$ for all $s \in X_{i} \cap S$ are connected. If Rule 5 removes $X_{i}$ from $G_{i}$, then $X_{i} \cap S=\emptyset$, i.e., 
$G^{r}\left[X_{i}^{\prime}\right]$ is a vertex-induced subgraph of the clique $G^{r}\left[X_{i}\right]$ and thus connected. If Rule $2 / 7$ removes $X_{i}$ from $G_{i}$, then $N_{G_{i}}\left(X_{i} \backslash S\right) \subseteq X_{i} \cap S$, i.e., each vertex $s^{\prime} \in S \cap X_{j}$ for $j>i$ is non-adjacent to $X_{i} \backslash S$ in $G^{r}$. Hence, at least one set $Y_{s}, s \in X_{i} \cap S$, contains a vertex $v$ from $X_{i} \backslash S$. The graph $G^{r}\left[\left(X_{i}^{\prime} \cap X_{i}\right) \backslash S\right]$ is a vertex-induced subgraph of the clique $G^{r}\left[X_{i} \backslash S\right]$ and thus connected. Hence, every vertex of $X_{i}^{\prime}$ is adjacent to $X_{i} \cap S=X_{i}^{\prime} \cap S$ or to $v$. Thus, $G^{r}\left[X_{i}^{\prime}\right]$ is connected.

Regarding $G_{i}^{\prime}$, we show that for every $v, v^{\prime} \in V\left(G_{i}^{\prime}\right)$ there is a $\left[v, v^{\prime}\right]$-path in $G_{i}^{\prime}$. The vertices $v$ and $v^{\prime}$ are also contained in $G_{i}$ because $G_{i}^{\prime}$ is a subgraph of $G_{i}$. Because $G_{i}$ is connected, there is a path $v=p_{1}, \ldots, p_{\ell}=v^{\prime}$ in $G_{i}$. If this path is not fully contained in $G_{i}^{\prime}$, let $j, j^{\prime} \in\{1, \ldots, \ell\}$ be indices such that $p_{j}, p_{j^{\prime}} \in V\left(G_{i}^{\prime}\right)$ and $p_{j+1}, \ldots, p_{j^{\prime}-1} \notin V\left(G_{i}^{\prime}\right)$. We show that there are vertices $w, w^{\prime} \in V\left(G_{i}^{\prime}\right)$ for which $G^{r}\left[\left\{p_{j}, w, w^{\prime}, p_{j^{\prime}}\right\}\right]$ is connected. This suffices to show the claim, as $G_{i}^{\prime}$ is a vertex-induced subgraph of $G^{r}$.

Because $G_{i}-$ Cand $=G_{i}^{\prime}$ - Cand, the vertices $p_{j+1}, \ldots, p_{j^{\prime}-1}$ must all be nonfixed internal vertices, and because they form a path, they must all belong to the same block $C$ of $G^{r}-S$. There are now two possibilities for $p_{j}$ and $p_{j^{\prime}}$ : Each of the two is either an external vertex of $C$ or contained in $S$. If $p_{j}$ is adjacent to every internal vertex of $C$, we can set $w:=p_{j}$.

Otherwise $p_{j} \in S$. Let $d$ be the index such that $p_{j} \in X_{d}$. Because $p_{j} \in V\left(G_{i}\right)$, it holds $d \geq i$. Furthermore, as $p_{j+1} \notin V\left(G_{i}^{\prime}\right)$, it holds $p_{j+1} \in X_{t}^{\prime}$ for some $t<i \leq d$. This means that $Y_{p_{j}}$ contains a vertex $w$ from $C_{\text {int }}$, for otherwise $p_{j+1}$ could have been added to $Y_{p_{j}}$. In the same way we can find a vertex $w^{\prime} \in\left(C_{\text {int }} \cap Y_{p_{j^{\prime}}}\right) \cup\left\{p_{j^{\prime}}\right\}$. In any case, $w, w^{\prime} \in V(C) \cap V\left(G_{i}^{\prime}\right)$, and $\left\{w, w^{\prime}\right\} \in E\left(G_{i}^{\prime}\right)$. This shows the claim. $\diamond$

Claim 4 The following inequalities hold for every $i \in\{0, \ldots, q-1\}$.

1. If $X_{i} \cap S \neq \emptyset$, then $\operatorname{ex}\left(G_{i}^{\prime}\right) \geq \operatorname{ex}\left(G_{i+1}^{\prime}\right)+\sum_{s \in X_{i}^{\prime} \cap S} \frac{\left|Y_{S} \backslash\{s\}\right|}{4}-1$.

2. If Rule 5 removes $X_{i}$ from $G_{i}$ with an anchor $w \in V\left(G_{i}\right)$, then

$$
\operatorname{ex}\left(G_{i}^{\prime}\right) \geq \operatorname{ex}\left(G_{i+1}^{\prime}\right)+\min \left\{\left|X_{i}^{\prime} \cap N^{+}(s)\right|,\left|X_{i}^{\prime} \cap N^{-}(s)\right|\right\}
$$

In particular, $\operatorname{ex}\left(G_{i}^{\prime}\right) \geq \operatorname{ex}\left(G_{i+1}^{\prime}\right)$ for the case that $w \notin S$.

Proof of the claim 1. First of all we know from Lemma 14 that $\operatorname{ex}\left(G\left[Y_{s}\right]\right) \geq \frac{\left|Y_{S} \backslash\{s\}\right|}{4}$ for every vertex $s \in X_{i}^{\prime} \cap S$. Furthermore, every subgraph $G\left[Y_{s}\right], s \in X_{i}^{\prime} \cap S$, is connected by construction. The set $X_{i}^{\prime} \backslash(\Gamma \cup S)$ is also connected because it is a vertex-induced subgraph of $X_{i} \backslash S$, which is a clique by the definitions of Rules 2/7. Hence, there is an ordering $Z_{1}, \ldots, Z_{d}$ of the sets $X_{i}^{\prime} \backslash(\Gamma \cup S)$ and $\left(Y_{S}\right)_{s \in S}$ such that every graph $\left(G\left[Z_{1} \cup \ldots \cup Z_{i}\right]\right)_{i \leq d}$ is connected. We can deduce from the definitions of Rules $1-7$ that $d \leq 4$. Hence, a $(d-1)$-fold application of Proposition 2 results in

$$
\operatorname{ex}\left(G_{i}^{\prime}\left[X_{i}^{\prime}\right]\right) \geq \sum_{s \in X_{i}^{\prime} \cap S} \frac{\left|Y_{s} \backslash\{s\}\right|}{4}-\frac{3}{4}
$$

Another application of the same proposition yields the first part of the claim. 
2. Like in the proof of Lemma 10, we use the fact that $G_{i}^{\prime}$ is a vertex-induced subgraph of $G_{i}$ with $G_{i}^{\prime}-$ Cand $=G_{i}-$ Cand. This means that the anchor $w$ of $X_{i}$ is also contained in $G_{i}^{\prime}$ and that $X_{i}^{\prime} \subseteq X_{i}$ cannot have any other neighbours than $w$. Then Rule 5 can remove $X_{i}^{\prime}$ from $G_{i}^{\prime}$ using $w$ as anchor. The second part of the claim follows now from Lemma 8.

For an index $i$ such that $X_{i}^{\prime}$ is removed by Rule 5 from $G_{i}^{\prime}$ using an anchor $s \in S$, define $\alpha_{i}:=\min \left\{\left|X_{i}^{\prime} \cap N^{+}(s)\right|,\left|X_{i}^{\prime} \cap N^{-}(s)\right|\right\}$ and $\beta_{i}:=\max \left\{\left|X_{i}^{\prime} \cap N^{+}(s)\right|, \mid X_{i}^{\prime} \cap\right.$ $\left.N^{-}(s) \mid\right\}$. Furthermore, let $\alpha_{s}$ be the sum of all $\alpha_{i}$ such that $s$ is the anchor of $X_{i}^{\prime}$, and let $\alpha:=\sum_{s \in S} \alpha$.

Because $X_{i} \cap S \neq \emptyset$ for at most $k$ many indices $i$ (otherwise, $\left(G^{r}, k\right)$ is a "yes"instance), the previous claim now shows that

$$
\operatorname{ex}\left(G^{r}\right)=\operatorname{ex}\left(G_{0}^{\prime}\right) \geq \sum_{s \in S} \frac{\left|Y_{s} \backslash\{s\}\right|}{4}-k+\alpha=\frac{|\Gamma|}{4}+\alpha-k
$$

Hence, if $\alpha \geq \frac{5 k}{4}$ or if $|\Gamma| \geq 5 k$, then $\operatorname{ex}\left(G^{r}\right) \geq \frac{k}{4}$ and $\left(G^{r}, k\right)$ is a "yes"-instance.

It remains to show that the total number of internal vertices in special blocks is bounded by $\Theta(|\Gamma|+\alpha)$. Let $B$ be a special block of $G^{r}-S$ and let $X=B_{\text {int }} \backslash(\Gamma \cup F)=$ ( $B_{\text {int }} \cap$ Cand $) \backslash \Gamma$ be the remaining candidate vertices in $B$. Let $i$ be the smallest index such that $V(B) \cap X_{i} \neq \emptyset$. There are the following possibilities.

1. The vertex set $X_{i}$ is removed by Rule $2 / 7$ from $G_{i}$ or $X_{i}$ is removed by Rule 5 using an anchor that is an external vertex of $G^{r}-S$. Then $V(B)=B_{\text {ext }} \cup\left(B_{\text {int }} \cap \Gamma\right) \cup X$, because if $B$ contains a fixed vertex, then this vertex must be an external vertex of $G^{r}-S$.

Suppose there were $x_{1}, x_{2} \in X$ such that there is a vertex $s \in\left(N^{+}\left(x_{1}\right) \backslash N^{+}\left(x_{2}\right)\right) \cap$ $S$. Then $x_{1}$ and $x_{2}$ could have been added to $Y_{s}$. (Note that $x_{1}$ and $x_{2}$ cannot be contained in $W_{s}$ because the anchor of $X_{i}$ is not in $S$.) The same argument for negative edges yields $N^{+}(x)=N^{+}(X)$ and $N^{-}(x)=N^{-}(X)$ for all $x \in X$.

Because Rule 8 cannot delete vertices from $B$, it now holds that

$$
|X| \leq \frac{|V(B)|+|N(X) \cap S|}{2}=\frac{\left|B_{\text {ext }}\right|+\left|B_{\text {int }} \cap \Gamma\right|+|X|+|N(X) \cap S|}{2},
$$

i.e., $|X| \leq\left|B_{\text {ext }}\right|+\left|B_{\text {int }} \cap \Gamma\right|+|N(X) \cap S|$. Now for every $s \in N(X) \cap S$ there is a vertex of $B_{\text {int }}$ in $Y_{s}$ (otherwise we could add an arbitrary vertex of $N(s) \cap X$ to it). Hence, $|N(X) \cap S| \leq\left|B_{\text {int }} \cap \Gamma\right|$ and the bound simplifies to $|X| \leq\left|B_{\text {ext }}\right|+$ $2 \cdot\left|B_{\text {int }} \cap \Gamma\right|$.

2. The vertex set $X_{i}$ is removed by Rule 5 using a vertex $w \in B_{\text {int }} \cap F$ as anchor.

The only difference to the case before is that $B_{\text {int }}$ now contains a (single) fixed vertex and thus $|V(B)|=\left|B_{\text {ext }}\right|+\left|B_{\text {int }} \cap \Gamma\right|+|X|+1$. The same reasoning results in the bound $|X| \leq\left|B_{\text {ext }}\right|+2 \cdot\left|B_{\text {int }} \cap \Gamma\right|+1$. Note that this case occurs only at most $|F| \leq k$ times.

3. The vertex set $X_{i}$ is removed by Rule 5 using a vertex $s \in S$ as anchor. Then $B$ does not contain a fixed vertex because we chose $i$ as the smallest index such 
that $X_{i}$ contains vertices from $B$, and an anchor from $s$ means that $G_{i+1}$ cannot contain vertices from $V(B)$ any more.

The difference to the cases before is that edges from $s$ to $X$ can be positive or negative, i.e., not all vertices of $X$ have the exactly same neighbourhood. But with the same arguments one shows that $N^{+}(x) \backslash\{s\}=N^{+}(X) \backslash\{s\}$ and $N^{-}(x) \backslash\{s\}=$ $N^{-}(X) \backslash\{s\}$ for all $x \in X$. Furthermore, because $X_{i}$ does not contain vertices from $S$, the set $X$ is identical to $X_{i}^{\prime}$, which has size $\left|X_{i}^{\prime}\right|=\alpha_{i}+\beta_{i}$.

Again due to Rule 8 we see that

$$
\begin{aligned}
& \qquad \beta_{i} \leq \frac{|V(B)|+|N(X) \cap S|}{2} \leq \frac{\left|B_{\text {ext }}\right|+\left|B_{\text {int }} \cap \Gamma\right|+\alpha_{i}+\beta_{i}+|N(X) \cap S|}{2}, \\
& \text { i.e., } \beta_{i} \leq\left|B_{\text {ext }}\right|+\left|B_{\text {int }} \cap \Gamma\right|+\alpha_{i}+|N(X) \cap S| \leq\left|B_{\text {ext }}\right|+2 \cdot\left|B_{\text {int }} \cap \Gamma\right|+\alpha_{i} \\
& \text { Then }|X|=\alpha_{i}+\beta_{i} \leq\left|B_{\text {ext }}\right|+2 \cdot\left|B_{\text {int }} \cap \Gamma\right|+2 \alpha_{i}
\end{aligned}
$$

Putting these bounds together shows that the number of internal vertices in special blocks can be bounded by

$$
\begin{aligned}
\sum_{B \in \mathcal{B}^{\star}}\left|B_{\text {int }}\right| & =\sum_{B \in \mathcal{B}^{\star}}\left(\left|B_{\text {int }} \cap F\right|+\left|B_{\text {int }} \cap \Gamma\right|+\left|B_{\text {int }} \backslash(F \cup \Gamma)\right|\right) \\
& \leq k+|\Gamma|+\sum_{B \in \mathcal{B}^{\star}}\left|B_{\text {int }} \backslash(F \cup \Gamma)\right| \\
& \leq k+|\Gamma|+\sum_{B \in \mathcal{B}^{\star}}\left(\left|B_{\text {ext }}\right|+2 \cdot\left|B_{\text {int }} \cap \Gamma\right|\right)+k+2 \alpha \\
& =\sum_{B \in \mathcal{B}^{\star}}\left|B_{\text {ext }}\right|+2 \cdot|\Gamma|+2 k+2 \alpha .
\end{aligned}
$$

Lemma 13 showed that $\sum_{B \in \mathcal{B}^{\star}}\left|B_{\text {ext }}\right| \leq 96 k$. Furthermore, we discussed already that $\alpha \leq \frac{5 k}{4}$ and $|\Gamma| \leq 5 k$ or $\left(G^{r}, k\right)$ is a "yes"-instance. Thus, the total number of internal vertices in special blocks is bounded from above by $96 k+10 k+2 k+\frac{5 k}{2} \leq$ $111 k$.

We are now ready to prove Theorem 2 .

Proof of Theorem 2 Let $\left(G^{0}, k\right)$ be an instance of SIGNED MAX-CUT AEE. Like in Sect. 3, apply Rules $1-7$ exhaustively to $\left(G^{0}, k\right)$ in time $O\left(k \cdot \mid E\left(G^{0} \mid\right)\right.$, producing an instance $\left(G^{\prime}, k^{\prime}\right)$ and a vertex set $S$ of marked vertices. If $k^{\prime} \leq 0$, then $\left(G^{\prime}, k^{\prime}\right)$ and thus also $(G, k)$ is a "yes"-instance.

Now apply Rules 8-9 exhaustively to $\left(G^{0}, k\right)$ in time $O(|E(G)|)($ Lemma 6) to obtain an equivalent instance $\left(G^{r}, k\right)$. Check whether $\left(G^{r}, k\right)$ is a "yes"-instance due to Lemma 13 or Lemma 15 . If this is not the case, then there are at most $3 k$ vertices in $S$, at most $48 k$ external vertices in $G^{r}-S$ and at most $111 k$ internal vertices in special blocks. If there were more internal than external vertices in a non-special block, we could apply Rule 8 to this block. Thus, the number of internal vertices in non-special blocks is bounded by $96 k$ according to Lemma 13 . Hence, the total number of vertices in $G^{r}$ is bounded by $3 k+48 k+111 k+96 k=258 k$. 


\section{Linear Vertex Kernels for $\lambda$-Extendible Properties}

In this section we extend our linear kernels for SIGNED MAX-CUT to all strongly $\lambda$-extendible properties satisfying (P1), or (P2), or (P3). Henceforth, fix a strongly $\lambda$ extendible property $\Pi$, and let $\left(G^{0}, k\right)$ be an instance of ABOve PolJAK-TurZíK $(\Pi)$. For notational brevity, we assume the empty graph to be in $\Pi$.

As in the previous section, we use a set of 1-safe reduction rules to find a set $S$ such that $G^{0}-S$ is a clique forest; the difference compared to SIGNED MAX-CUT is the different change of $k$. These rules were initially devised by Mnich et al. [30]; for sake of completeness, we list them here. Every rule takes an instance $(G, k)$ and produces an instance $\left(G^{\prime}, k^{\prime}\right)$ such that $(G, k)$ is a "yes"-instance if $\left(G^{\prime}, k^{\prime}\right)$ is. Initially, $S:=\emptyset$.

Reduction Rule 10 Let $v \in V(G)$ and $C$ be a connected component of $G-v$ such that $G[V(C) \cup\{v\}]$ is a clique. Delete $C$ from $G$ and set $k^{\prime}=k$.

Reduction Rule 11 Suppose Rule 10 does not apply. Let $C_{1}, \ldots, C_{\ell}$ be the connected components of $G-v$ for some vertex $v \in V(G)$. If at least one of the $C_{i} s$ is a clique, and at most one of them is not a clique, then add $v$ to $S$, delete $v$ and all the $C_{i} s$ which are cliques from $G$, and set $k^{\prime}=k-d \cdot \frac{1-\lambda}{2}$, where $d$ is the number of deleted cliques.

Reduction Rule 12 For vertices $a, b, c \in V(G)$ inducing a path $(a, b, c)$ such that $G-\{a, b, c\}$ is connected, add $a, b, c$ to $S$, delete them from $G$, and set $k^{\prime}=$ $k-\frac{1-\lambda}{2}$.

Reduction Rule 13 Suppose Rule 12 does not apply. Let $v, b \in V(G)$ such that $\{v, b\} \notin E(G)$. Let $C_{1}, \ldots, C_{\ell}$ be the connected components of $G-\{v, b\}$. If there is at least one $C_{i}$ such that both $G\left[V\left(C_{i}\right) \cup\{v\}\right]$ and $G\left[V\left(C_{i}\right) \cup\{v\}\right]$ are cliques, and there is at most one $C_{i}$ for which this does not hold, then add $v, b$ to $S$, delete them from $G$, delete all the $C_{i} s$ which satisfy the conditions, and set $k^{\prime}=k-\frac{1-\lambda}{2}$.

Proposition 6 [30, Lemmas 6-8] Rules 10-13 are 1-safe and can each be applied in polynomial time. To any connected graph with at least one edge, one of these rules applies and the resulting graph is connected. The exhaustive application of the rules to $\left(G^{0}, k\right)$ either decides that $\operatorname{ex}\left(G^{0}\right) \geq k$, or finds a set $S$ of at most $\frac{6 k}{1-\lambda}$ vertices such that $G^{0}-S$ is a clique forest. This also holds for all strongly $\lambda$-extendible properties of oriented and/or labelled graphs.

The detection which of the reduction rules can be applied to a graph $G$ is completely analogous to the SIGNED MAX- CUT reduction rules. Hence, it follows immediately from Lemma 3 that the rules can be applied exhaustively in time $O(\mathrm{~km})$.

\subsection{Linear Kernel for Properties Diverging on Cliques}

We first show that ABOve POLJAK-TuRzík $\operatorname{BOUND}(\Pi)$ admits kernels with $O(k)$ vertices for all strongly $\lambda$-extendible properties $\Pi$ that are diverging on cliques and for which $\operatorname{ex}\left(K_{i}\right)>0$ for all $i \geq 2$. 
For this subsection, let $\left(G^{0}=G_{0}, \ldots, G_{q}\right)$ be the sequence of graphs generated by the exhaustive application of Rules $10-13$ to $\left(G^{0}, k\right)$, let $S$ be the set of marked vertices, and let $X_{0}, \ldots, X_{q-1}$ be vertex sets such that $G_{i}-X_{i}=G_{i+1}$ for all $i<q$.

Definition 5 Let $i<q$ be an index such that Rule 10 removes $X_{i}$ from $G_{i}$. Let $w \in$ $V\left(G_{i+1}\right)$ be anchor of $X_{i}$. We call the vertices from $X_{i}$ bad if $\operatorname{ex}\left(G\left[X_{i} \cup\{w\}\right]\right)=0$. We also call the block of $G^{0}-S$ containing $X_{i}$ bad.

Lemma 16 Let $\Pi$ be a strongly $\lambda$-extendible property diverging on cliques, and let $\left(G^{0}, k\right)$ be an instance of ABOVE POLJAK-TURZí $(\Pi)$. Let $\Gamma$ be the set of bad vertices resulting from an exhaustive application of Rules 10-13 to $\left(G^{r}, k\right)$. Then $\left|V\left(G^{0}\right) \backslash \Gamma\right|=O(k)$ or $\left(G^{r}, k\right)$ is a "yes"-instance.

As a consequence, if $\operatorname{ex}\left(K_{i}\right)>0$ for all $i \geq 2$, then $\operatorname{ABOVE} \operatorname{POLJAK}-\operatorname{TuRZíK}(\Pi)$ admits a kernel with $O(k)$ vertices.

Proof We know already that $|S|=O(k)$ or $\left(G^{0}, k\right)$ is a "yes"-instance. If $\operatorname{ex}\left(K_{i}\right)>0$ for all $i \geq 2$, then there are no bad vertices, i.e., $|\Gamma|=0$. In the following we show that there is a constant $\varepsilon>0$ such that $\operatorname{ex}\left(G_{i}\right) \geq \operatorname{ex}\left(G_{i+1}\right)+\varepsilon \cdot\left|X_{i} \backslash(S \cup \Gamma)\right|$ for every index $i<q$. This implies that $|V(G) \backslash(S \cup \Gamma)|=O(k)$ or $\left(G^{0}, k\right)$ is a "yes"-instance, showing the lemma.

Because $\Pi$ diverges on cliques, there is by definition an integer $j \in \mathbb{N}$ and a constant $a>0$ such that $\operatorname{ex}\left(K_{j}\right)=\frac{1-\lambda}{2}+a$. Note that $j$ only depends on $\Pi$ and not the instance $\left(G^{0}, k\right)$. Hence, we can treat $j$ as constant for a given property $\Pi$. Let

$$
\tau^{\prime}:=\min \left\{\frac{\operatorname{ex}(G)}{|V(G)|} \mid \operatorname{ex}(G)>0 \text { and }\langle G\rangle=K_{i} \text { for some } 2 \leq i<j\right\} \text {. }
$$

Then $\tau^{\prime}$ is well-defined, since $j$ is constant; moreover, it can be computed in polynomial time by computing the excess of all graphs $G$ such that $\langle G\rangle$ is a clique of size up to $j$. Furthermore, $\tau^{\prime}>0$ holds by definition.

Let $\tau:=\min \left\{\tau^{\prime}, \frac{a}{2 j}\right\}>0$.

Claim 5 Let $C$ be a clique with $|V(C)| \geq\left(a+3 \cdot \frac{1-\lambda}{2}\right) \cdot\left(\frac{a}{j}-\tau\right)^{-1}=: M$. Then $\operatorname{ex}(C) \geq$ $\tau \cdot|V(C)|+3 \cdot \frac{1-\lambda}{2}$.

Proof Let $i:=|V(C)|$, and let $r:=\left\lfloor\frac{i}{j}\right\rfloor$. Then Proposition 1 assures that $\operatorname{ex}(C) \geq r \cdot a$. This means that

$$
\operatorname{ex}(C) \geq r a \geq \frac{i}{j} \cdot a-a \geq 3 \cdot \frac{1-\lambda}{2}+\tau \cdot i
$$

where the last inequality holds if

$$
i \cdot\left(\frac{a}{j}-\tau\right) \geq a+3 \cdot \frac{1-\lambda}{2} .
$$

This is exactly the bound for $i=|V(C)|$ given in the claim. Note that $\frac{a}{j}-\tau>0$ by the definition of $\tau$. 
Let now $\varepsilon:=\min \left\{\frac{\tau}{2}, \frac{1-\lambda}{2 M}\right\}>0$.

Claim 6 For every $i<q$, it holds $\operatorname{ex}\left(G_{i}\right) \geq \operatorname{ex}\left(G_{i+1}\right)+\varepsilon \cdot\left|X_{i} \backslash S\right|$.

Proof of the claim Let $i<q$. We consider the following different cases.

1. Rule 12 removes $X_{i}$ from $G_{i}$. Then there is nothing to show, as $X_{i} \backslash S=\emptyset$.

2. Rule 10 removes $X_{i}$ from $G_{i}$. Let $C$ and $v$ be defined like in Rule 10, i.e., $X_{i}=$ $V(C), C$ is a connected component of $G_{i}-v$, and $B:=G_{i}[V(C) \cup\{v\}]$ is a clique of size at least 2. If $C$ contains bad vertices, there is again nothing to show. Assume therefore from now on that $V(C) \cap \Gamma=\emptyset$. This means that $\operatorname{ex}(B)>0$.

Because $\left|X_{i} \backslash S\right|=|V(B)|-1 \geq|V(B)| / 2$ and because $B$ is a block of $G_{i}$, by the block additivity of $\Pi$ it suffices to show that $\operatorname{ex}(B) \geq \tau \cdot|V(B)|$.

If $|V(B)|<j$, this is immediately clear. Otherwise, let $r:=\left\lfloor\frac{|V(B)|}{j}\right\rfloor$. Proposition 1 assures that $\operatorname{ex}(B) \geq r \cdot a$, whereas $|V(B)| \leq(r+1) \cdot j$. Because $r \geq 1$, we therefore obtain $\frac{\operatorname{ex}(\bar{B})}{|V(B)|} \geq \frac{r \cdot a}{(r+1) \cdot j} \geq \frac{a}{2 j} \geq \tau$.

3. Rule 13 removes $X_{i}$ from $G_{i}$. Let $v, b \in X_{i} \cap S$. Mnich et al. [30, Observation 19] showed that $X_{i}$ consists of $v, b$, and a single clique $C$. If $|V(C)| \geq M$, then $\operatorname{ex}(C) \geq \tau \cdot|V(C)|+3 \cdot \frac{1-\lambda}{2}$. Proposition 1 then shows

$$
\begin{aligned}
\operatorname{ex}\left(G_{i}\left[X_{i}\right]\right) & \geq \operatorname{ex}\left(G_{i}\left[X_{i} \backslash\{v\}\right]\right)-\frac{1-\lambda}{2} \geq \operatorname{ex}\left(G_{i}\left[X_{i} \backslash\{v, b\}\right]\right)-2 \cdot \frac{1-\lambda}{2} \\
& \geq \tau \cdot|V(C)|+\frac{1-\lambda}{2}
\end{aligned}
$$

Another application yields

$$
\operatorname{ex}\left(G_{i}\right)-\operatorname{ex}\left(G_{i+1}\right) \geq \operatorname{ex}\left(G_{i}\left[X_{i}\right]\right)-\frac{1-\lambda}{2} \geq \tau \cdot|V(C)| \geq \varepsilon \cdot|V(C)|
$$

Otherwise, $|V(C)| \leq M$. Because Rule 13 is 1-safe, it holds

$$
\operatorname{ex}\left(G_{i}\right)-\operatorname{ex}\left(G_{i+1}\right) \geq \frac{1-\lambda}{2} \geq \frac{1-\lambda}{2 M} \cdot|V(C)| \geq \varepsilon \cdot|V(C)|
$$

4. Rule 11 removes $X_{i}$ from $G_{i}$. Let $C_{1}, \ldots, C_{d}$ be the connected components of $G_{i}\left[X_{i} \backslash S\right]$. Then every $C_{i}$ is a clique. Note that $d$ is exactly the variable named $d$ in the definition of Rule 11 .

Order the cliques so that $\left|V\left(C_{j}\right)\right| \geq M$ if and only if $j \leq p$ for some $p \in$ $\{0, \ldots, d\}$.

If $p=0$, then, as Rule 11 is 1 -safe, it holds

$$
\operatorname{ex}\left(G_{i}\right)-\operatorname{ex}\left(G_{i+1}\right) \geq d \cdot \frac{1-\lambda}{2} \geq d \cdot \frac{1-\lambda}{2 d M} \cdot\left|X_{i} \backslash S\right| \geq \varepsilon \cdot\left|X_{i} \backslash S\right|
$$


Consider now the case $p>0$. The graph $G^{\prime}:=G-\bigcup_{j \leq p} V\left(C_{j}\right)$ is still connected. Then the previous claim together with Proposition 2 yields

$$
\operatorname{ex}\left(G_{i}\right) \geq \operatorname{ex}\left(G^{\prime}\right)+\sum_{j=1}^{p} \tau \cdot\left|V\left(C_{j}\right)\right|+2 p \cdot \frac{1-\lambda}{2} \geq \operatorname{ex}\left(G^{\prime}\right)+\sum_{j=1}^{p} \tau \cdot\left|V\left(C_{j}\right)\right|+\frac{1-\lambda}{2}
$$

Now if $C_{1}, \ldots, C_{p}$ did not exist, then Rule $11 / 13$ could still remove $C_{p+1}, \ldots, C_{d}$, decreasing $k$ by $(d-p) \cdot \frac{1-\lambda}{2} \geq \varepsilon \cdot \sum_{j>p}\left|V\left(C_{j}\right)\right|$. Now observe that

$$
\operatorname{ex}\left(G_{i}-\left(X_{i} \backslash S\right)\right) \geq \operatorname{ex}\left(G_{i+1}\right)-\frac{1-\lambda}{2}
$$

because $X_{i} \cap S$ consists of a single vertex. Putting these bounds together yields the desired result $\operatorname{ex}\left(G_{i}\right) \geq \operatorname{ex}\left(G_{i+1}\right)+\varepsilon \cdot\left|X_{i} \backslash S\right|$.

The lemma follows now immediately from the previous claim.

Theorem 4 Let $\Pi$ be a strongly $\lambda$-extendible property. If $\lambda \neq \frac{1}{2}$ or $G \in \Pi$ for every $G$ with $\langle G\rangle=K_{3}$, then ABOVE POLJAK-TURZíK $(\Pi)$ admits a kernel with $O(k)$ vertices.

Proof Crowston et al. [9, Lemmas 24-26] show that if $\lambda \neq \frac{1}{2}$ or $K_{3} \in \Pi$, then $\Pi$ diverges on cliques and $\operatorname{ex}\left(K_{i}\right)>0$ for all $i \geq 2$. Therefore, we can apply Lemma 16 .

\subsection{Strongly $\frac{1}{2}$-Extendible Properties on Oriented Graphs}

We now turn to strongly $\frac{1}{2}$-extendible properties $\Pi$ on oriented graphs. We can now use a subset of Rules 1-7 again, to be more precise, exactly the rules that are applicable to signed graphs with only negative edges. This has the advantage that we will be able to reuse Lemma 7.

We restate the rules here because the parameter $k$ is scaled by a factor of $\frac{1}{4}$ due to the different problem definitions. Let $G$ always be a connected graph.

Reduction Rule 14 Let $C$ be a connected component of $G-v$ for some vertex $v \in$ $V(G)$ such that $G[V(C) \cup\{v\}]$ is a clique. Delete $C$ and set $k^{\prime}=k$.

Reduction Rule 15 Let $C$ be a connected component of $G-v$ for some vertex $v \in$ $V(G)$ such that $C$ is a clique. If there exist $a, b \in V(C)$ such that $G-\{a, b\}$ is connected and $\{a, v\} \in E(G)$, but $\{b, v\} \notin E(G)$, then add $a, b$ to $S$ and delete them from $G$, and set $k^{\prime}=k-\frac{1}{2}$.

Reduction Rule 16 Let $(a, b, c)$ be an induced 2-path for some $a, b, c \in V(G)$ such that $G-\{a, b, c\}$ is connected. Add $a, b, c$ to $S$ and delete them from $G$, and set $k^{\prime}=$ $k-\frac{1}{4}$. 
Reduction Rule 17 Let $v, b \in V(G)$ such that $\{v, b\} \notin E(G)$ and $G-\{v, b\}$ has exactly two connected components $C$, Y. If $G[V(C) \cup\{v\}]$ and $G[V(C) \cup\{b\}]$ are cliques, then add $v, b$ to $S$ and delete them from $G$ together with $C$, and set $k^{\prime}=k-\frac{1}{4}$.

Rules 14-17 are exactly Rules 5/3/6/7 for SIGNED MAX- CUT AEE with all edges negative.

Note that the concept of bad vertices translates naturally to vertices removed by Rule 14, which is equivalent to Rule 10.

Lemma 17 Rules 14-17 are 1-safe. To any connected graph with at least one edge, one of the rules applies and the resulting graph is connected. If $S$ is the set of marked vertices, then $G-S$ is a clique forest. If $|S|>12 k$, then $(G, k)$ is a "yes"-instance.

Proof First note that we cannot deduce 1-safeness from the fact that these rules are 1-safe for Signed MAX-CUT AEE. But Rules 14/16/17 are 1-safe because they are equal to or special cases of Rules 10-13, which were shown to be 1-safe for all $\lambda$-extendible properties.

Now we show that Rule 15 is 1 -safe. Let $G, a, b, v$ and $C$ like in the description of the rule, and let $G^{\prime}$ be the resulting graph. Let $H^{\prime}$ be a $\Pi$-subgraph of $G^{\prime}$. We extend $H^{\prime}$ to a $\Pi$-subgraph $H$ of $G$ by adding the vertices $a, b$, the edge $\{a, b\}$, and at least $\left\lceil\frac{\left|E\left(\{a, b\}, V\left(G^{\prime}\right)\right)\right|}{2}\right\rceil$ edges between $\{a, b\}$ and $V\left(G^{\prime}\right)$. We can do this due to the extendibility property of $\Pi$. Now observe that $\left|E\left(\{a, b\}, V\left(G^{\prime}\right)\right)\right|$ is odd because every vertex of $V\left(G^{\prime}\right) \backslash\{v\}$ is adjacent to $a$ if and only if it is adjacent to $b$. Therefore, we can guarantee that

$$
\begin{aligned}
|E(H)| & \geq\left|E\left(H^{\prime}\right)\right|+1+\left\lceil\frac{\left|E\left(\{a, b\}, V\left(G^{\prime}\right)\right)\right|}{2}\right\rceil \\
& =\left|E\left(H^{\prime}\right)\right|+1+\frac{\left|E\left(\{a, b\}, V\left(G^{\prime}\right)\right)\right|+1}{2} .
\end{aligned}
$$

As $\operatorname{pt}(G)=\operatorname{pt}\left(G^{\prime}\right)+\frac{2}{4}+\frac{1}{2}+\frac{\left|E\left(\{a, b\}, V\left(G^{\prime}\right)\right)\right|}{2}$, it follows that $\operatorname{ex}(G) \geq \operatorname{ex}\left(G^{\prime}\right)+\frac{1}{2}$, i.e., Rule 15 is 1 -safe.

Now we argue that one of the rules applies to any connected graph $G$ with at least one edge. Let $G^{\prime}$ be the signed graph that results from adding a negative sign to every edge of $\langle G\rangle$. We know from Proposition 5 that one of the Rules 1-7 applies to $G^{\prime}$. To be more precise, Rule 3/5/6/7 applies to $G^{\prime}$ because all other rules require at least one positive edge. But the mentioned rules correspond exactly to Rules 14-17, i.e., one of these rules must apply to $G$.

Let $H$ be the resulting graph after applying one of the Rules 14-17 to $G$, and let $H^{\prime}$ be the resulting graph after applying the corresponding rule of the Rules $3 / 5 / 6 / 7$ to $G^{\prime}$. Then $\left\langle G^{\prime}\right\rangle=\left\langle H^{\prime}\right\rangle$ and the same set of vertices has been marked. Therefore, we can follow from Proposition 5 that $H$ is connected and that $G-S$ is a clique forest.

The last claim simply follows from the fact that Rules $14-17$ decrease $k$ by at least $\frac{1}{12}$ for every vertex that is added to $S$. Note that the change from $|S| \geq 3 k$ to $|S| \geq 12 k$ stems from the different meanings of $k$ : in the SIGNED MAX-CUT AEE problem the question is whether $\operatorname{ex}(G) \geq \frac{k}{4}$, whereas ABOvE POLJAK-TuRZík $(\Pi)$ asks whether $\operatorname{ex}(G) \geq k$. 
Like Crowston et al. [9], we restrict ourselves to hereditary properties. Let $\vec{K}_{3}$ be the orientation of $K_{3}$ which is an oriented cycle, and let $\vec{K}_{3}$ be the only (up to isomorphisms) other orientation of $K_{3}$. Crowston et al. [9] showed that if $\vec{K}_{3} \in \Pi$, then also $\vec{K}_{3} \in \Pi$, and thus Theorem 4 applies. We now consider the case that $\vec{K}_{3} \notin \Pi$ together with $\vec{K}_{3} \in \Pi$.

Proposition 7 [9] Let $\Pi$ be a hereditary strongly $\frac{1}{2}$-extendible property on oriented graphs with $\vec{K}_{3} \in \Pi$. Then $\operatorname{ex}\left(K_{i}\right)>0$ for all $i \geq 4$ and $\Pi$ diverges on cliques.

Following Proposition 7, Lemma 16 shows a "kernel" with $O(k)+|\Gamma|$ vertices, where $\Gamma$ is the set of bad vertices of an input graph. The only oriented clique with at least two vertices, but without positive excess is $\vec{K}_{3}$, because $\operatorname{ex}\left(K_{2}\right)=\frac{1}{4}$ for $\frac{1}{2}$ extendible properties. Therefore, a vertex is bad if and only if it belongs to a set $X$ removed from $G$ by Rule 14 using an anchor $w$ such that $G[X \cup\{w\}] \cong \vec{K}_{3}$. Hence, we only need reduction rules to bound the number of blocks $B$ in a clique forest with $B \cong \vec{K}_{3}$. (Note: If the anchor $w$ is contained in $S$, then the resulting block in the clique forest has only two vertices, but this can happen only once per connected component of $G^{r}-S$, the number of which is dominated by other tractable terms.)

Let $\Pi$ be a hereditary strongly $\frac{1}{2}$-extendible property on oriented graphs with $\vec{K}_{3} \in \Pi$. Let $\left(G^{0}, k\right)$ be an instance of ABOve POLJAK-TURZíK $(\Pi)$. Lemma 17 either proves that $\left(G^{0}, k\right)$ is a "yes"-instance, or it finds a set $S$ of at most $12 k$ vertices such that $G^{0}-S$ is a clique forest. Starting with $\left(G^{0}, k\right)$, we apply the following reduction rules, which on input $(G, k)$ produce an equivalent instance $\left(G^{\prime}, k\right)$.

Reduction Rule 18 Let $a, b \in V(G) \backslash S$ and $w \in V(G) \backslash\{a, b\}$ such that $N_{G}(a)=$ $\{w, b\}, N_{G}(b)=\{w, a\}$, and $G[\{w, a, b\}] \cong \vec{K}_{3}$. Delete $a$ and $b$ from $G$.

Reduction Rule 19 Let $B_{1}, B_{2}, B_{3}$ be non-leaf-blocks in $G-S$ and let $v_{1}, \ldots, v_{4} \in$ $V(G)$ be such that

- $v_{i}, v_{i+1} \in\left(B_{i}\right)_{\text {ext }}$ for all $i \in\{1,2,3\}$;

$-B_{i} \cong \vec{K}_{3}$ for all $i \in\{1,2,3\}$; and

- $N_{G}\left(\left\{v_{2}, v_{3}, w_{1}, w_{2}, w_{3}\right\}\right)=\left\{v_{1}, v_{4}\right\}$, where $w_{i}$ is the internal vertex of $B_{i}$.

Delete $v_{3}, w_{3}$, and add edges $\left\{v_{2}, v_{4}\right\}$ and $\left\{w_{2}, v_{4}\right\}$ to $G$.

Intuitively speaking, Rule 19 takes three blocks in $G-S$ that form a "path" and are all isomorphic to $\vec{K}_{3}$. If all vertices except the "endpoints" $v_{1}$ and $v_{4}$ are not adjacent to $S$ and not contained in other blocks from $G-S$, then it is safe to delete one block. For an illustration, see Fig. 2.

Lemma 18 Let $\Pi$ be a hereditary strongly $\frac{1}{2}$-extendible property on oriented graphs with $\vec{K}_{3} \in \Pi$. Then Rules 18-19 are 2-safe. The resulting graphs are connected. 
Fig. 2 Illustration of Rule 19
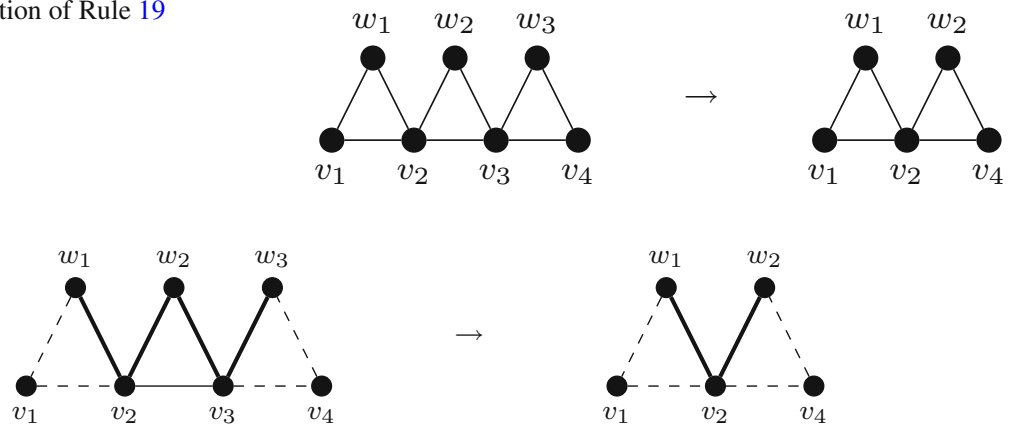

Fig. 3 The $\Pi$-subgraph $Y$ in $G$ and the $\Pi$-subgraph $Y^{\prime}$ in $G^{\prime}$ are highlighted by thick edges. At least half of the dotted edges between $\tilde{H}$ and $Y$ or $Y^{\prime}$, respectively, can be added to a $\Pi$-subgraph

Proof Let $G^{\prime}$ be the resulting graph. Then $G^{\prime}$ is clearly connected. Rule 18 is 2safe because $\operatorname{ex}(G)$ is additive on 2-connected components and $G[\{a, b, w\}]$ is a 2-connected component of $G$.

To show that Rule 19 is 2 -safe, let $\widetilde{G}=G-\left\{v_{2}, v_{3}, w_{1}, w_{2}, w_{3}\right\}$. Let $X:=V\left(B_{1}\right) \cup$ $V\left(B_{2}\right) \cup V\left(B_{3}\right)$ and $X^{\prime}:=X \cap V\left(G^{\prime}\right)$. First note that $|E(H[X])| \leq 6$ for any $\Pi$ subgraph $H$ of $G$ and $\left|E\left(H\left[X^{\prime}\right]\right)\right| \leq 4$ for any $\Pi$-subgraph $H$ of $G^{\prime}$, for otherwise there would be a block $B_{i}$ with $\left|E\left(H\left[V\left(B_{i}\right)\right]\right)\right|=3$. This is a contradiction, as $\Pi$ is hereditary and $\vec{K}_{3} \notin \Pi$. Also note that $\operatorname{pt}(\underset{\widetilde{G}}{G})=\operatorname{pt}\left(G^{\prime}\right)+2$.

As $\Pi$ is hereditary, it holds that $H[V(\widetilde{G})] \in \Pi$ and $H^{\prime}[V(\widetilde{G})] \in \Pi$ for all $\Pi$ subgraphs $H$ and $H^{\prime}$ of $G$ and $G^{\prime}$. Hence, it suffices to show that we can extend every $\Pi$-subgraph $\widetilde{H}$ of $\widetilde{G}$ to $\Pi$-subgraphs $H$ and $H^{\prime}$ of $G$ and $G^{\prime}$ such that $|E(H[X])|=6$ and $\left|E\left(H\left[X^{\prime}\right]\right)\right|=4$.

Let $\widetilde{H}$ be a $\Pi$-subgraph of $\widetilde{G}$. Let $Y=G\left[X \backslash\left\{v_{1}, v_{4}\right\}\right]-\left\{v_{2} v_{3}\right\}$ and $Y^{\prime}=G^{\prime}\left[X^{\prime} \backslash\right.$ $\left.\left\{v_{1}, v_{4}\right\}\right]$. Examine Fig. 2 again for an illustration. Then $Y$ and $Y^{\prime}$ both are trees with 4 and 2 edges, respectively, and hence $Y, Y^{\prime} \in \Pi$; see Fig. 3 .

By the extendibility of strongly $\frac{1}{2}$-extendible properties, there is a $\Pi$-subgraph $H$ of $G$ which contains all edges of $\widetilde{H}$, all edges of $Y$ and at least half of the edges between them. There are exactly 4 edges between them $\widetilde{H}$ and $Y$, at most two of which can be contained in $H$ as otherwise $\vec{K}_{3}$ would be a subgraph of $H$. Hence, $|E(H[X])|=6$. With the same arguments we obtain a $\Pi$-subgraph $H^{\prime}$ of $G^{\prime}$ with $\left|E\left(H^{\prime}\left[X^{\prime}\right]\right)\right|=4$; this completes the proof.

From now on, let $G^{r}$ be the resulting graph after the exhaustive application of Rules 18-19 on $G^{0}$. Furthermore, let $\left(G^{r}=G_{0}, \ldots, G_{q}\right)$ be the sequence of graphs generated by the exhaustive application of Rules $14-17$ to $\left(G^{r}, k\right)$, let $S$ be the set of marked vertices, and let $X_{0}, \ldots, X_{q-1}$ be the vertex sets such that $G_{i}-X_{i}=G_{i+1}$ for every $i<q$.

Rules 18-19 are special cases of Rules 8-9. As Rules 14-17 are Rules 5/3/6/7 for SIGNED MAX-CUT AEE with all edges negative, the next lemma follows from Lemma 7. 
Lemma 19 Rules 14-17 can be applied exhaustively on the graph $G^{r}$ in such a way that the set $S^{\prime}$ of vertices removed by their application is equal to $S$.

Let $\mathcal{B}^{-}$be the set of bad blocks in $G^{r}-S$ and let $\mathcal{B}^{+}$be the set of all other blocks in $G^{r}-S$. Let $\tilde{\mathcal{B}}$ be the set of bad special blocks, i.e., the set of blocks $B \in \mathcal{B}$ with $B_{\text {int }} \cap N_{G^{r}}(S) \neq \emptyset$. Furthermore, let $R$ be the set of vertices $r \in V\left(G^{r}\right) \backslash S$ such that

1. $r$ is contained in exactly two blocks $B_{1}, B_{2}$ of $G^{r}-S$,

2. both blocks $B_{1}$ and $B_{2}$ are bad,

3. $\left(B_{1}\right)_{\text {int }} \neq \emptyset \neq\left(B_{2}\right)_{\text {int }}$, and

4. $B_{1}$ and $B_{2}$ are not special.

Lemma 20 It holds $\left|\mathcal{B}^{-}\right|=O\left(\left|\mathcal{B}^{+}\right|+|\tilde{\mathcal{B}}|+|R|\right)$.

Proof Let $V^{-}:=\bigcup_{B \in \mathcal{B}^{-}} V(B)$ be the set of vertices contained in bad blocks (i.e., the bad vertices together with anchors that are not in $S$ and used to removed bad vertices), and let $H:=G\left[V^{-}\right]$. Because $V^{-} \subseteq V\left(G^{r}\right) \backslash S$ and because $G^{r}-S$ is a clique forest, also the graph $H$ is a clique forest.

Consider now a block forest $F$ of $H$ (see Definition 4). For a block $B$ of $H$, denote the corresponding vertex in $F$ by $f_{B}$. Let $n_{1}, n_{2}$, and $n_{\geq 3}$ be the number of vertices in $F$ with degree 1, 2, and at least 3, respectively. Every leaf $f_{B}$ of $F$ corresponds to a leaf block $B$ in $H$, i.e., $n_{1}$ is at most the number of leaf blocks in $H$. Therefore we first bound the number of leaf blocks in $H$.

Claim 7 The number of leaf blocks in $H$ is bounded by $O\left(\left|\mathcal{B}^{+}\right|+|\tilde{\mathcal{B}}|\right)$.

Proof of the claim Let $B$ be a leaf block of $H$. Then there are the following possibilities:

- $B$ is an isolated block in $G^{r}-S$. Let $i$ be the index such that $X_{i}$ contains two vertices of $B$. Then $X_{i}$ was removed by Rule 14 using an anchor $w \in V(G)$ with $G^{r}[V(B) \cup\{w\}] \cong \vec{K}_{3}$. Because $B$ did not get eliminated by Rule 18 , it must hold that $N_{G^{r}}(V(B)) \backslash\{w\} \neq \emptyset$. This means that $B$ is special. There can only be at most $|\tilde{\mathcal{B}}|$ many of these blocks.

$-|V(B)|=2$ and $B$ is isolated in $H$, but not in $G^{r}-S$. This means that $B$ shares a vertex $v$ with a block from $\mathcal{B}^{+}$. This vertex $v$ is not contained in any other block from $\mathcal{B}^{-}$. Therefore, there can only be at most $\left|\mathcal{B}^{+}\right|$many of these blocks.

$-|V(B)|=2$ and $B$ is not isolated in $H$. Then $B$ shares an external vertex with a block $B^{\prime}$ in $H$. Because every connected component of $H$ can only contain at most one block with exactly two vertices (namely, the block removed last), the number of such blocks $B$ is bounded by the number of blocks in $H$ with three vertices.

$-|V(B)|=3$. Then $B$ contains three vertices $u, v, w$ such that $N_{H}(u)=\{v, w\}$ and $N_{H}(v)=\{u, w\}$. Because $u$ and $v$ did not get eliminated by Rule 18 , there must be a vertex $z \in V(G)$ such that $z \in N_{G^{r}}(\{u, v\})$. If $z \in S$, then $B$ is special. Otherwise, $B$ shares a vertex with a block from $\mathcal{B}^{+}$, and this vertex is not contained in any other block from $\mathcal{B}^{-}$. Therefore the total number of blocks $B$ with $|V(B)|=3$ is bounded by $|\tilde{\mathcal{B}}|+\left|\mathcal{B}^{+}\right|$. 
Because $F$ is a forest, it holds $n_{\geq 3} \leq n_{1}$. It now suffices to bound $n_{2}$ because the number of blocks in $H$ is equal to $n_{1}+n_{2}+n_{\geq 3}$.

Let $f_{B_{1}}$ and $f_{B_{2}}$ be two adjacent vertices in $F$. There are the following possibilities.

- The degrees of both $f_{B_{1}}$ and $f_{B_{2}}$ are not equal to 2 . The number of such pairs is already bounded by $O\left(n_{1}\right)$.

- $\left|N_{H}\left(f_{B_{1}}\right)\right|=2$ and $\left|N_{H}\left(f_{B_{2}}\right)\right|=1$. There are at most $n_{1}$ such pairs.

- $\left|N_{H}\left(f_{B_{1}}\right)\right|=2$ and $\left|N_{H}\left(f_{B_{2}}\right)\right| \geq 3$. There are at most $n_{1}+n_{\geq 3} \leq 2 n_{1}$ such pairs. One can easily see this by contracting all vertex sets $X$ of $F$ such that $X$ is a path and $\left|N_{H}(x)\right|=2$ for all $x \in X$.

- $\left|N_{H}\left(f_{B_{1}}\right)\right|=2$ and $\left|N_{H}\left(f_{B_{2}}\right)\right|=2$. The number of cases where $B_{1}$ or $B_{2}$ is a leaf block (this can happen if this block is selected as root of the connected component of $F$ in the definition of block forests) is bounded by the number of leaf blocks in $H$.

The number of cases that $B_{1}$ or $B_{2}$ contains only two vertices is bounded by the number of connected components of $H$, which is in turn bounded by the number of leaf blocks in $H$.

It remains the case that $B_{1}$ and $B_{2}$ are not leaf blocks and $B_{1}$ and $B_{2}$ both contain exactly 3 vertices. Then both blocks each contain exactly one internal vertex. The number of cases where $B_{1}$ or $B_{2}$ is special is bounded by $|\tilde{\mathcal{B}}|$.

If this is not the case, let $w$ be the external vertex shared by $B_{1}$ and $B_{2}$. Then $w \in R$. Hence, the number of these remaining pairs is bounded by $|R|$.

This concludes the proof.

Lemma 21 It holds $\left|\mathcal{B}^{+}\right|+|\tilde{\mathcal{B}}|=O(k)$ or $\left(G^{r}, k\right)$ is a "yes"-instance.

Proof Let $B \in \mathcal{B}^{+}$and let $i<q$ be the index that generates $B$. If Rule 15-17 removes $X_{i}$ from $G_{i}$, then $\operatorname{ex}\left(G_{i}\right) \geq \operatorname{ex}\left(G_{i+1}\right)+\frac{1}{4}$ because these rules are 1-safe.

On the other hand, if Rule 14 removes $X_{i}$ from $G_{i}$ using an anchor $w \in V\left(G_{i}\right)$, then $\operatorname{ex}\left(G_{i}\left[X_{i} \cup\{w\}\right]\right)>0$ because $B$ is not a bad block. Because $\lambda=\frac{1}{2}$, the term $4 \cdot \operatorname{ex}(G)$ must be integral for every graph $G$. It follows from the block additivity of $\Pi$ that $\operatorname{ex}\left(G_{i}\right)-\operatorname{ex}\left(G_{i+1}\right) \geq \operatorname{ex}\left(G_{i}\left[X_{i} \cup\{w\}\right]\right) \geq \frac{1}{4}$. Hence, $\left|\mathcal{B}^{+}\right| \leq 4 k$.

We now turn to the number of bad special blocks. Because Rules 14-17 is exactly the subset of Rules 1-7 applicable to signed graphs without positive edges, and because the kernelization Rules 8-9 for SIGNED MAX-CUT AEE and Rules 18-19 for ABOVE POLJAK-TURZíK $(\Pi)$ do not change the number of special blocks, we can derive from Lemma 10 that the number of special blocks in $G^{r}-S$ is bounded by $O(k)$ or $\left(G^{r}, k\right)$ is a "yes"-instance.

Lemma 22 It holds $|R|=O\left(\left|R \cap N_{G^{r}}(S)\right|\right)$. Furthermore, it holds $\left|R \cap N_{G^{r}}(S)\right|=$ $O\left(\left|\mathcal{B}^{+}\right|+|\tilde{\mathcal{B}}|+k\right)$ or $\left(G^{r}, k\right)$ is a "yes"-instance.

Proof For the first part of the proof, let $r_{1}, r_{2} \in R$ be adjacent in $G^{r}$. Then there are by the definition of $R$ three blocks $B_{1}, B_{2}, B_{2} \in \mathcal{B}^{-}$such that $r_{1} \in V\left(B_{1}\right)$, $r_{1}, r_{2} \in V\left(B_{2}\right)$, and $r_{2} \in V\left(B_{3}\right)$, such that $B_{1}, B_{2}, B_{3}$ are bad and each have an internal vertex that is not adjacent to $S$. Furthermore, there are no other blocks of $G^{r}-S$ containing $r_{1}$ or $r_{2}$. 
Fig. 4 An example for the sets $T$ and $U$. The vertices $t_{1}, t_{2}$ are in $T$, the

vertices $t_{1}^{\prime}, t_{1}^{\prime \prime}, t_{2}^{\prime}, t_{2}^{\prime \prime}$ are the corresponding internal vertices in $U$, and $s_{1}, s_{2}$ are in $S$. Edges contained in $G[U]$ are bold

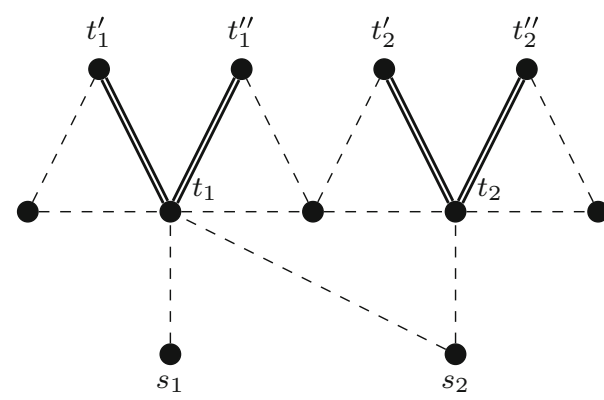

If both $r_{1}$ and $r_{2}$ were not adjacent to $S$, then all conditions of Rule 19 would be met ( $r_{1}$ and $r_{2}$ would correspond to $v_{2}$ and $v_{3}$ in the definition of this rule). Hence, at least one of $r_{1}$ and $r_{2}$ is adjacent to $S$. Because every vertex of $R$ is adjacent to at most two other vertices from $R$, this means that $|R|=O\left(\left|R \cap N_{G^{r}}(S)\right|\right)$.

Now we turn to the second part of the lemma. Let $T$ be a maximum independent set in $G\left[R \cap N_{G^{r}}(S)\right]$. Again, as every vertex of $R$ is adjacent to at most two other vertices from $R$, it holds $|T| \geq \frac{1}{3} \cdot\left|R \cap N_{G^{r}}(S)\right|$.

Every vertex $t \in T \subseteq R$ is by definition of $R$ adjacent to exactly two internal vertices $t^{\prime}, t^{\prime \prime}$ of $G^{r}-S$, and these two vertices are not adjacent to $S$. For $t \in T$, let $U_{t}=\left\{t, t^{\prime}, t^{\prime \prime}\right\}$, and let $U:=\bigcup_{t \in T}\left\{t, t^{\prime}, t^{\prime \prime}\right\}$. Then $G^{r}[U]$ is a forest, becaue if there was an internal vertex $x$ from $G^{r}-S$ adjacent to two vertices $t_{1}, t_{2} \in T$, then $t_{1}$ and $t_{2}$ would be adjacent. See Fig. 4 for an illustration.

Because every vertex of $T$ is adjacent to $S$, there is a set $\left(Y_{S}\right)_{S \in S}$ of stars with the following properties:

- Every star $Y_{s}$ is a vertex-induced subgraph of $G^{r}$ centered in $s$ and its leafs are from $T$.

- $\bigcup_{s \in S} V\left(Y_{s}\right)$ covers $T$.

Now we enhance the stars $Y_{s}$ by replacing every leaf $t$ with $U_{t}$, i.e., we define $Z_{s}$ to be the subgraph of $G^{r}$ induced by $s$ and $U_{t}$ for every $t \in T$ that is a leaf of $Y_{s}$. Then every $Z_{s}, s \in S$, is a forest with $\left|E\left(Z_{s}\right)\right|=3 \cdot\left|E\left(Y_{s}\right)\right|=3 \cdot\left|V\left(Y_{s}\right) \cap T\right|$ many edges. By the block additivity of $\Pi$, it follows $\operatorname{ex}\left(Z_{S}\right)=\frac{3 \cdot\left|V\left(Y_{S}\right) \cap T\right|}{4}$ for every $s \in S$.

Repeated application of Proposition 2 yields

$$
\operatorname{ex}(G[Z]) \geq \sum_{s \in S} \frac{3 \cdot\left|V\left(Y_{S}\right) \cap T\right|}{4}-\frac{|S|}{4}=\frac{3 \cdot|T|-|S|}{4}
$$

where $Z:=\bigcup_{s \in S} V\left(Z_{S}\right)$. This means that

$$
\operatorname{ex}\left(G^{r}\right) \geq \operatorname{ex}(G[Z])-\frac{c+1}{4} \geq \frac{3 \cdot|T|-|S|-c-1}{4}
$$


where $c$ is the number of connected components of $G^{\prime}:=G-Z$. Now it suffices to show that $c \leq|T|+\left|\mathcal{B}^{+}\right|+|\tilde{\mathcal{B}}|$, since then

$$
\operatorname{ex}\left(G^{r}\right) \geq \frac{2 \cdot|T|-\left|\mathcal{B}^{+}\right|-|\tilde{\mathcal{B}}|-O(k)}{4},
$$

i.e., $|T|=O\left(\left|\mathcal{B}^{+}\right|+|\tilde{\mathcal{B}}|+k\right)$ or $\left(G^{r}, k\right)$ is a "yes"-instance.

Let us first bound the number of connected components of $G^{r}-S$. It is clear that $G^{r}-S$ can only have $\left|\mathcal{B}^{+}\right|$many connected components that contain a block that is not bad. Let now $W$ be a connected component of $G^{r}-S$ that contains only bad blocks. If $W$ contains only one block, then this block is special. Otherwise, $W$ contains at least two leaf blocks, and because only one of the (bad) blocks of $W$ can have size 2 , there is a leaf block $B$ in $W$ with $|V(B)|=3$. Because $B$ was not eliminated by Rule 18, it must be special. Hence, the number of connected components of $G^{r}-S$ containing only bad blocks is bounded by $|\tilde{\mathcal{B}}|$ and thus the total number of connected components of $G^{r}-S$ is bounded by $\left|\mathcal{B}^{+}\right|+|\tilde{\mathcal{B}}|$.

Because every vertex from $U \backslash T$ is an internal vertex in $G^{r}-S$, the removal of these vertices from $G^{r}-S$ cannot increase the number of connected components. Furthermore, as every vertex from $T$ is contained in exactly two blocks of $G^{r}-S$, its removal can increase the number of connected components by at most 1 . Hence, the number $c$ of connected components of $G-Z$ is at most the number of connected components of $G-S$ plus $|T|$. This completes the proof.

Theorem 5 Let $\Pi$ be a hereditary strongly $\frac{1}{2}$-extendible property on oriented graphs with $\vec{K}_{3} \in \Pi$. Then ABOvE POLJAK-TURZíK $(\Pi)$ admits a kernel with $O(k)$ vertices.

Proof Let $\left(G^{0}, k\right)$ be an instance of ABOve PolJAK-TurzíK $(\Pi)$. Apply Rules $14-$ 17 exhaustively, producing an instance $\left(G^{\prime}, k^{\prime}\right)$ and a vertex set $S$. If $k^{\prime} \leq 0$, then $\left(G^{\prime}, k^{\prime}\right)$ and thus also $\left(G^{0}, k\right)$ is a "yes"-instance. Otherwise, $|S|=O(k)$.

Now apply Rules $18-19$ exhaustively on $\left(G^{0}, k\right)$ to obtain an equivalent instance $\left(G^{r}, k\right)$. Let $\Gamma$ be the set of bad vertices of $G^{r}$. Lemma 16 shows that $\left|V\left(G^{r}\right) \backslash \Gamma\right|=$ $O(k)$ or $\left(G^{r}, k\right)$ is a "yes"-instance. Because every bad vertex is in a bad block and every bad block contains at most three vertices, it holds $|\Gamma|=O\left(\left|\mathcal{B}^{-}\right|\right)$. Using Lemma 20, Lemma 21, and Lemma 22, we can bound this cardinality by

$$
\left|\mathcal{B}^{-}\right|=O\left(\left|\mathcal{B}^{+}\right|+|\tilde{\mathcal{B}}|+|R|\right)=O\left(\left|\mathcal{B}^{+}\right|+|\tilde{\mathcal{B}}|+k\right)=O(k)
$$

or $\left(G^{r}, k\right)$ is a "yes"-instance. This shows the theorem.

We are ready to complete the proof of Theorem 3 .

Proof of Theorem 3 Let $\lambda \in(0,1)$ and let $\Pi$ be a strongly $\lambda$-extendible property of (possibly oriented and/or labelled) graphs. If $\lambda \neq \frac{1}{2}$ or $G \in \Pi$ for every $G$ with $\langle G\rangle=K_{3}$, we can use Theorem 4. Otherwise, we only have to consider the case that $\Pi$ is a hereditary property of simple or oriented graphs.

Consider the case that $\vec{K}_{3} \in \Pi$ or $\vec{K}_{3} \in \Pi$. If $\vec{K}_{3} \in \Pi$, then Crowston et al. [9] show that $\vec{K}_{3} \in \Pi$, i.e., we can use Theorem 4 . And if $\vec{K}_{3} \in \Pi$, we use Theorem 5. 
Now we may suppose that $G \notin \Pi$ for every $G$ with $\langle G\rangle=K_{3}$. Then Crowston et al. [9] show that $\Pi$ is the set of all bipartite graphs. Hence, in the case of simple graphs as well as if $\vec{K}_{3}, \vec{K}_{3} \notin \Pi$ for oriented graphs, we can use Theorem 2 to obtain a linear vertex kernel.

It is easy to see that Rules $18-19$ can be applied exhaustively in time $O(m)$. As $\lambda$ is constant and we can apply every other reduction rule in linear time, it follows a total run time of $O(\lambda \cdot \mathrm{km})=O(\mathrm{~km})$.

\section{Discussion}

For the classical (SIGNED) MAX- CUT problem, and its wide generalization to strongly $\lambda$-extendible properties, parameterized above the classical Poljak-Turzík bound, we improved the run time analysis for a known fixed-parameter algorithm to $8^{k} \cdot O(m)$. We further improved all known kernels with $O\left(k^{3}\right)$ vertices for these problems to asymptotically optimal $O(k)$ vertices. We did not try to optimize the hidden constants, as the analysis is already quite cumbersome.

A natural question to ask is whether this problem admits faster algorithms and smaller kernels, say with run time $2^{k} \cdot O(m)$ and $2 k$ vertices respectively, or whether such results can be ruled out assuming a standard hypothesis.

It remains an interesting question whether all positive results presented here extend to edge-weighted graphs, where each edge receives a positive integer weight and the number $m$ of edges in the Edwards-Erdôs bound (1) is replaced by the total sum of the edge weights.

Further, Mnich et al. [30] showed fixed-parameter tractability of ABOVE POLJAK$\operatorname{TURZíK}(\Pi)$ for all strongly $\lambda$-extendible properties $\Pi$. However, the polynomial kernelization results by Crowston et al. [9] as well as in this paper do not seem to apply to the special case of non-hereditary $\frac{1}{2}$-extendible properties. Such properties $\Pi$ exist; e.g., $\Pi=\left\{G \in \mathcal{G} \mid C \nRightarrow K_{3}\right.$ for all 2-connected components $C$ of $\left.G\right\}$. Also, for $\frac{1}{2}$ extendible properties on labelled graphs we only showed a polynomial kernel for the special case of SIGNED MAX- CUT. It would be desirable to avoid these restrictions.

Acknowledgements We thank the anonymous reviewers for their feedback, which helped us to improve the presentation of our results.

Open Access This article is distributed under the terms of the Creative Commons Attribution 4.0 International License (http://creativecommons.org/licenses/by/4.0/), which permits unrestricted use, distribution, and reproduction in any medium, provided you give appropriate credit to the original author(s) and the source, provide a link to the Creative Commons license, and indicate if changes were made.

\section{References}

1. Alon, N., Gutin, G., Kim, E.J., Szeider, S., Yeo, A.: Solving MAX-r-SAT above a tight lower bound. Algorithmica 61(3), 638-655 (2011)

2. Barahona, F.: On the computational complexity of Ising spin glass models. J. Phys. A 15(10), 32413253 (1982)

3. Bollobás, B., Scott, A.: Better bounds for max cut. In: Bollobás, B. (ed.) Contemporary Combinatorics, Bolyai Society Mathematical Studies, vol. 10, pp. 185-246. Springer, Berlin (2002) 
4. Chiang, C., Kahng, A.B., Sinha, S., Xu, X., Zelikovsky, A.Z.: Fast and efficient bright-field AAPSM conflict detection and correction. IEEE Trans. Comput. Aided Des. Integr. Circuits Syst. 26(1), 115-126 (2007)

5. Crowston, R., Fellows, M., Gutin, G., Jones, M., Kim, E.J., Rosamond, F., Ruzsa, I.Z., Thomassé, S., Yeo, A.: Satisfying more than half of a system of linear equations over GF(2): a multivariate approach. J. Comput. Syst. Sci. 80(4), 687-696 (2014)

6. Crowston, R., Gutin, G., Jones, M.: Directed acyclic subgraph problem parameterized above the PoljakTurzík bound. In: Proceedings of FSTTCS 2012, Leibniz International Proceedings in Informatics, vol. 18, pp. 400-411, Hyderabad (2012)

7. Crowston, R., Gutin, G., Jones, M., Muciaccia, G.: Maximum balanced subgraph problem parameterized above lower bound. Theor. Comput. Sci. 513, 53-64 (2013)

8. Crowston, R., Jones, M., Mnich, M.: Max-cut parameterized above the Edwards-Erdôs bound. Algorithmica 72(3), 734-757 (2015)

9. Crowston, R., Jones, M., Muciaccia, G., Philip, G., Rai, A., Saurabh, S.: Polynomial kernels for $\lambda$ extendible properties parameterized above the Poljak-Turzík bound. In: Proceedings of FSTTCS 2013, Leibniz International Proceedings in Informatics, vol. 24, pp. 43-54, Guwahati (2013)

10. Dorn, F.: Planar subgraph isomorphism revisited. In: Proceedings of STACS 2010, Leibniz International Proceedings in Informatics, vol. 5, pp. 263-274 (2010)

11. Dvořák, Z., Mnich, M.: Large independent sets in triangle-free planar graphs. SIAM J. Discrete Math. 31(2), 1355-1373 (2017)

12. Edwards, C.S.: Some extremal properties of bipartite subgraphs. Can. J. Math. 25, 475-485 (1973)

13. Edwards, C.S.: An improved lower bound for the number of edges in a largest bipartite subgraph. In: Recent Advances in Graph Theory, pp. 167-181 (1975)

14. Etscheid, M., Mnich, M.: Linear kernels and linear-time algorithms for finding large cuts. In: Proceedings of ISAAC 2016, Leibniz Int. Proc. Informatics, vol. 64, pp. 31:1-31:13 (2016)

15. Gutin, G., van Iersel, L., Mnich, M., Yeo, A.: Every ternary permutation constraint satisfaction problem parameterized above average has a kernel with a quadratic number of variables. J. Comput. Syst. Sci. 78(1), 151-163 (2012)

16. Gutin, G., Rafiey, A., Szeider, S., Yeo, A.: The linear arrangement problem parameterized above guaranteed value. Theory Comput. Syst. 41(3), 521-538 (2007)

17. Gutin, G., Yeo, A.: Note on maximal bisection above tight lower bound. Inf. Process. Lett. 110(21), 966-969 (2010)

18. Harary, F.: On the notion of balance of a signed graph (1953-54). Mich. Math. J. 2, 143-146 (1955)

19. Harary, F.: On the measurement of structural balance. Behav. Sci. 4, 316-323 (1959)

20. Harary, F., Lim, M.H., Wunsch, D.C.: Signed graphs for portfolio analysis in risk management. IMA J. Manag. Math. 13(3), 201-210 (2002)

21. Hüffner, F., Betzler, N., Niedermeier, R.: Separator-based data reduction for signed graph balancing. J. Comb. Optim. 20(4), 335-360 (2010)

22. Impagliazzo, R., Paturi, R., Zane, F.: Which problems have strongly exponential complexity? J. Comput. Syst. Sci. 63(4), 512-530 (2001)

23. Iwata, Y., Wahlström, M., Yoshida, Y.: Half-integrality, LP-branching, and FPT algorithms. SIAM J. Comput. 45(4), 1377-1411 (2016)

24. Karp, R.M.: Reducibility among combinatorial problems. In: Complexity of computer computations (Proc. Sympos., IBM Thomas J. Watson Res. Center, Yorktown Heights, NY, 1972), pp. 85-103. Plenum, New York (1972)

25. Knuutila, T.: Re-describing an algorithm by Hopcroft. Theor. Comput. Sci. 250(1-2), 333-363 (2001)

26. König, D.: Über Graphen und ihre Anwendung auf Determinantentheorie und Mengenlehre. Math. Ann. 77(4), 453-465 (1916)

27. Lokshtanov, D., Ramanujan, M.S., Saurabh, S.: Linear time parameterized algorithms for subset feedback vertex set. In: Proceedings of ICALP 2015. Lecture Notes Computer Science, vol. 9134, pp. 935-946 (2015)

28. Mahajan, M., Raman, V.: Parameterizing above guaranteed values: MaxSat and MaxCut. Tech. Rep. TR97-033, Electronic Colloquium on Computational Complexity (1997). http://eccc.hpi-web. de/report/1997/033/

29. Mahajan, M., Raman, V., Sikdar, S.: Parameterizing above or below guaranteed values. J. Comput. Syst. Sci. 75(2), 137-153 (2009) 
30. Mnich, M., Philip, G., Saurabh, S., Suchý, O.: Beyond Max-Cut: $\lambda$-extendible properties parameterized above the Poljak-Turzík bound. J. Comput. Syst. Sci. 80(7), 1384-1403 (2014)

31. Ngọc, N.V., Tuza, Z.: Linear-time approximation algorithms for the max cut problem. Comb. Probab. Comput. 2(2), 201-210 (1993)

32. Poljak, S., Turzík, D.: A polynomial time heuristic for certain subgraph optimization problems with guaranteed worst case bound. Discrete Math. 58(1), 99-104 (1986)

33. Poljak, S., Tuza, Z.: Maximum cuts and large bipartite subgraphs. In: Combinatorial Optimization (New Brunswick, NJ, 1992-1993), DIMACS Series in Discrete Mathematics Theoretical Computer Science, vol. 20, pp. 181-244 (1995)

34. Raman, V., Saurabh, S.: Parameterized algorithms for feedback set problems and their duals in tournaments. Theor. Comput. Sci. 351(3), 446-458 (2006)

35. Raman, V., Saurabh, S.: Improved fixed parameter tractable algorithms for two "edge" problems: MAXCUT and MAXDAG. Inf. Process. Lett. 104(2), 65-72 (2007)

36. van Bevern, R.: Fixed-parameter linear-time algorithms for NP-hard graph and hypergraph problems arising in industrial applications. Ph.D. thesis, TU Berlin (2014) 